\title{
DESENVOLVIMENTO DE UM CÓDIGO COMPUTACIONAL DE APOIO AO CÁLCULO DE DOSE INTERNA PARA RADIONUCLÍDEOS DE INTERESSE DO IPEN
}

\section{Thiago Ribeiro Claro}

\author{
Dissertação apresentada como parte dos \\ requisitos para obtenção do Grau de \\ Mestre em Ciências na Área de Tecnologia \\ Nuclear - Aplicações \\ Orientador: \\ Dr. Alberto Saburo Todo
}

São Paulo

2011 
Ao meu filho Lucas, assim como todas as outras coisas que fiz nos últimos 10 anos. 
Agradecimentos

Ao amigo Dr. Alberto Saburo Todo, pelo apoio e orientação na execução deste trabalho.

Ao amigo Eduardo Heredia, pela ajuda e incentivo na conclusão do trabalho.

Ao amigo Dr. Goro Hiromoto, por compartilhar sua paciência e sabedoria.

Ao ex-aluno e hoje grande amigo Luiz Rodrigo Martins Barbosa, pela grande contribuição tecnológica.

Ao grupo de radioproteção do IPEN e todos seus funcionários que direta ou indiretamente colaboraram neste trabalho. 


\title{
Desenvolvimento de um código computacional de apoio ao cálculo de dose interna para radionuclídeos de interesse do IPEN.
}

\section{Thiago Ribeiro Claro}

\begin{abstract}
RESUMO
A dose resultante da contaminação interna pode ser estimada por meio de modelos biocinéticos em conjunto com os resultados experimentais obtidos de medidas de bioanálise e do conhecimento do momento da incorporação. Os modelos biocinéticos são representados por um conjunto de compartimentos que expressam o transporte, a retenção e a eliminação dos radionuclídeos do organismo. As publicações 66, 78 e 100 da ICRP apresentam modelos compartimentais para o trato respiratório, trato gastrointestinal e de distribuição sistêmica para diversos radionuclídeos de interesse para a proteção radiológica. O objetivo deste trabalho é desenvolver um código computacional para utilização em Dosimetria Interna considerando os principais radionuclídeos de interesse do IPEN do ponto de vista das exposições ocupacionais, tanto em situações rotineiras como também em casos acidentais, de modo a servir de ferramenta ágil e eficiente na construção, visualização e resolução de modelos compartimentais de qualquer natureza. A arquitetura do sistema foi concebida contendo dois programas independentes: CBT - responsável pela criação e manipulação dos modelos, e SSID - responsável pela resolução matemática dos mesmos. São oferecidas quatro técnicas diferentes para a resolução do sistema de equações incluindo métodos semi-analíticos e numéricos, onde se pode comparar a precisão e o desempenho destas. O desenvolvimento foi feito na linguagem de programação C\#, utilizando um banco de dados Microsoft Access e o padrão XML para a troca de arquivos com outros aplicativos. Para a validação do programa CBT foram construídos os modelos de compartimentos para os radionuclídeos urânio, tório e iodo. Com o programa SSID os modelos foram resolvidos e os resultados comparados com os valores publicados pela ICRP 78. Em todos os casos, o sistema desenvolvido conseguiu reproduzir os valores publicados pela ICRP.
\end{abstract}




\title{
Development of a computational code for the internal doses assessment of the main radionuclides of occupational exposure at IPEN.
}

\author{
Thiago Ribeiro Claro
}

\begin{abstract}
The dose resulting from internal contamination can be estimated with the use of biokinetic models combined with experimental results obtained from bioanalysis and assessment of the time of incorporation. The biokinetics models are represented by a set of compartments expressing the transportation, retention and elimination of radionuclides from the body. The ICRP publications, number 66, 78 and 100, present compartmental models for the respiratory tract, gastrointestinal tract and for systemic distribution for an array of radionuclides of interest for the radiological protection. The objective of this work is to develop a computational code for the internal doses assessment of the main radionuclides of occupational exposure at IPEN. Consequently serving as a agile and efficient tool for the designing, visualization and resolution of compartmental models of any nature. The architecture of the system was conceived containing two independent software: CBT - responsible for the setup and manipulation of models and SSID - responsible for the mathematical solution of the models. Four different techniques are offered for the resolution of system of equations, including semi-analytical and numerical methods, allowing for comparison of precision and performance of both. The software was developed in C\# programming, using a Microsoft Access database and XML standards for file exchange with other applications. Compartmental models for uranium, thorium and iodine radionuclides were generated for the validation of the CBT software. The models were subsequently solved via SSID software and the results compared with the values published in the issue 78 of ICRP. In all cases the system replicated the values published by ICRP.
\end{abstract}




\section{SUMÁRIO}

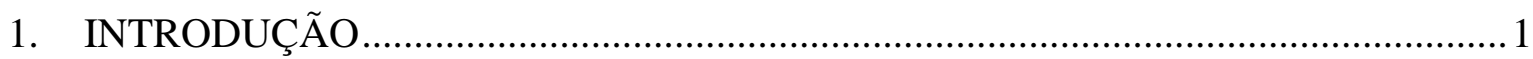

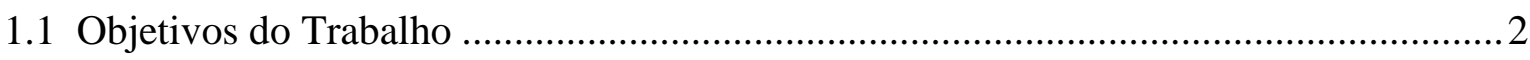

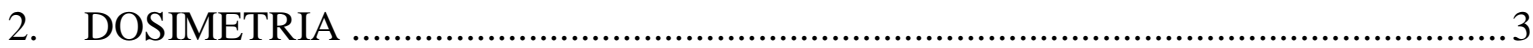

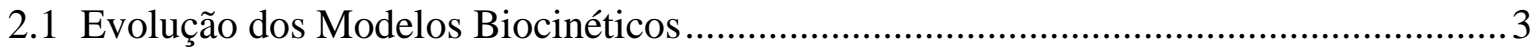

2.2 Metodologia da ICRP para Avaliação da Dose ……...................................................

2.3 Revisão dos métodos e códigos computacionais disponíveis ......................................... 6

2.4 Programa de monitoração individual interna do IPEN ...............................................

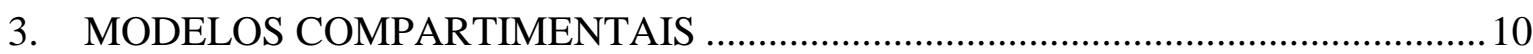

3.1 Modelos para a representação da incorporação, transferência e liberação do material.

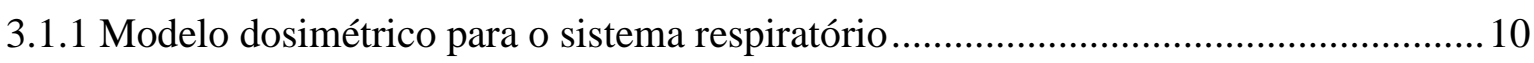

3.1.2 Modelo dosimétrico para o sistema gastrointestinal ................................................. 14

3.2 Descrição matemática dos modelos compartimentais .................................................. 16

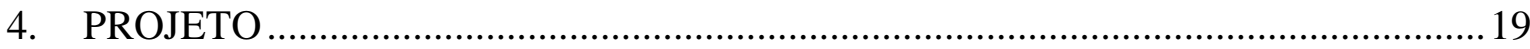

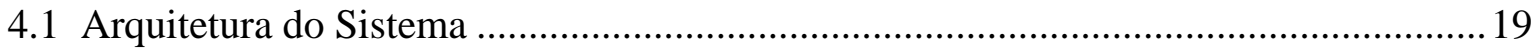

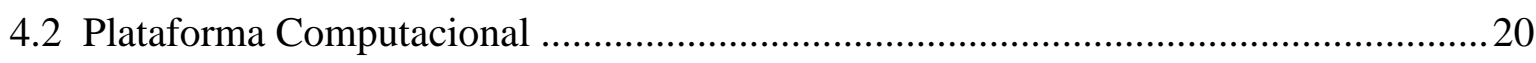

4.3 Compartiment Builder Tool - CBT ...................................................................... 21

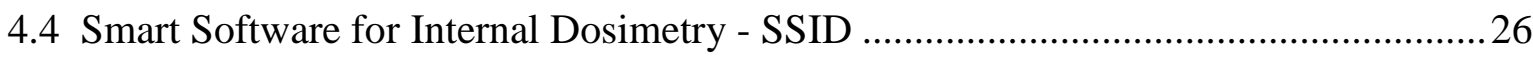

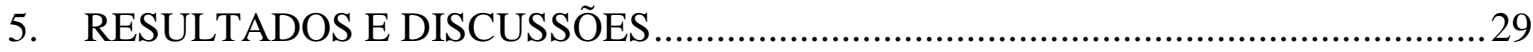

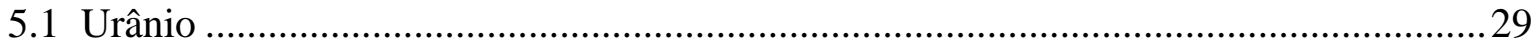

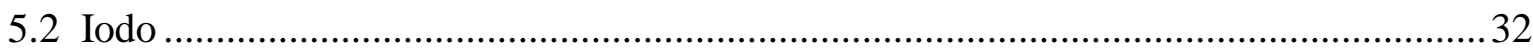

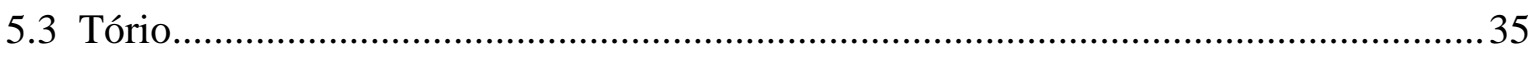

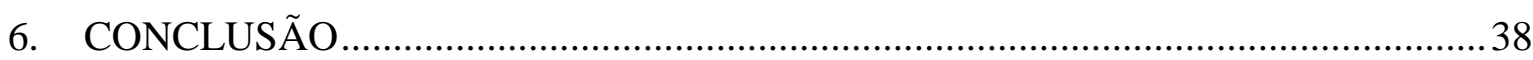

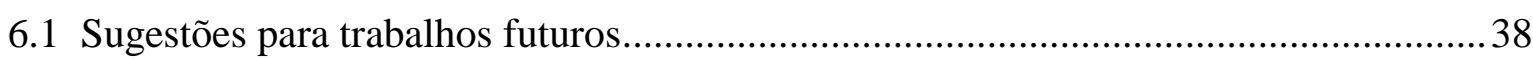

APÊNDICE A - Exemplo de XML gerado pelo CBT ….................................................. 40

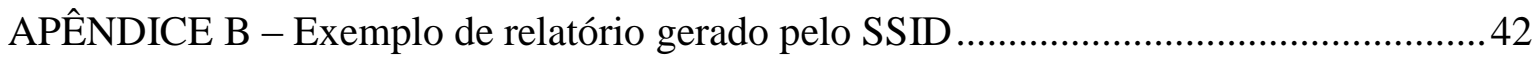

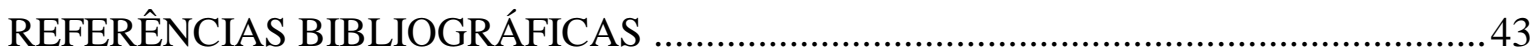




\section{Lista de Figuras}

FIGURA 1 Diagrama do programa de monitoração interna........................................ 8

FIGURA 2 Regiões do sistema respiratório humano................................................ 11

FIGURA 3 Modelo compartimental descrevendo o transporte de partículas em cada região do trato respiratório.

FIGURA 4 Representação da dissolução de um composto do tipo $\mathrm{S}$ em função do tempo no trato respiratório.

FIGURA 5 Modelo matemático que descreve a cinética dos radionuclídeos no trato gastrointestinal apresentado pela ICRP 30.

FIGURA 6 Modelo do Trato Alimentar Humano apresentado pela ICRP 100........ 16

FIGURA 7 Arquitetura do sistema desenvolvido.................................................... 19

FIGURA $8 \quad$ Modelo de banco de dados utilizado...................................................... 22

FIGURA 9 Tela de escolha do modelo a ser utilizado.............................................. 22

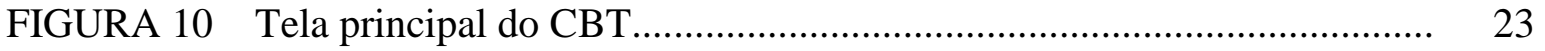

FIGURA 11 Detalhe da tela do CBT exibindo as ligações....................................... 24

FIGURA 12 Compartimentos relacionados que apresentam realimentação................ 25

FIGURA 13 Tela principal do SSID após a resolução de um modelo........................ 27

FIGURA 14 Gráfico gerado pelo SSID após a resolução de um modelo..................... 28

FIGURA 15 Diagrama do modelo biocinético do urânio, adaptado da ICRP 78........ 31

FIGURA 16 Modelo sistêmico para o metabolismo do iodo, adaptado da ICRP 78... 33

FIGURA 17 Curvas de retenção do sangue e da tireóide para o Iodo, gerado pelo SSID.

FIGURA 18 Gráfico comparativo entre os resultados da ICRP e o SSID para o Iodo

FIGURA 19 Diagrama do modelo biocinético para tório, plutônio, amerício, cúrio e netúnio. Adaptado da ICRP 78

FIGURA 20 Gráfico com os Valores de retenção e excreção do Tório 228 gerado pelo SSID 


\section{INTRODUÇÃO}

O IPEN - Instituto de Pesquisas Energéticas e Nucleares - possui instalações onde diariamente trabalhadores manipulam materiais radioativos. Uma parte destas atividades envolve o manuseio de fontes não seladas, onde existe um risco de contaminações e incorporações de tais materiais apesar dos procedimentos da Proteção Radiológica. As principais vias de incorporação de radionuclídeos são a inalação, a ingestão e a incorporação através da pele.

A quantidade de um radionuclídeo incorporada por um indivíduo pode ser avaliada diretamente ou indiretamente. Técnicas diretas, chamadas de in vivo, procuram avaliar a atividade presente no organismo utilizando detectores de radiação conhecidos como detectores de corpo inteiro. Medidas in vitro avaliam a quantidade do radionuclídeo presente no organismo por meio de análises de amostras biológicas, como urina ou fezes sendo consideradas técnicas indiretas. Entretanto, os resultados das medidas experimentais, isoladamente, não permitem a avaliação das doses resultantes desta incorporação.

A ICRP (International Commission on Radiological Protection - Comissão Internacional de Proteção Radiológica) regularmente publica recomendações para os limites de doses para trabalhadores, e modelos biocinéticos que descrevem o comportamento de determinados radionuclídeos no corpo humano.

A dosimetria interna combina informações da Proteção Radiológica, dos modelos biocinéticos e das medidas experimentais, com o objetivo de se estimar a atividade incorporada e conseqüentemente avaliar as doses recebidas pelos trabalhadores ocupacionalmente expostos.

Fundamentados no conjunto de recomendações da ICRP, diversos códigos computacionais foram desenvolvidos com o intuito de auxiliar na determinação da atividade incorporada. Eles são baseados na resolução do sistema de equações diferenciais que surge da representação dos modelos biocinéticos por um conjunto de compartimentos.

No entanto, dada as características peculiares das instalações do IPEN, tais códigos necessitam de atualizações para a inclusão de novos modelos publicados pela 
ICRP, tornando-os onerosos e trabalhosos. Além disso, muitos destes programas computacionais não estão atualizados com os novos modelos dosimétricos e biocinéticos.

\subsection{Objetivos do Trabalho}

O presente trabalho tem como objetivo o desenvolvimento de um código computacional para a construção, visualização e resolução de modelos compartimentais permitindo a inclusão de novos radionuclídeos e alterações nos modelos já existentes.

Os objetivos específicos são:

- Implementação computacional de diferentes técnicas matemáticas de resolução de sistemas de equações diferenciais para a solução dos modelos compartimentais.

- Elaboração de um programa computacional para a construção de modelos compartimentais tanto para a representação dos modelos biocinéticos quanto para a representação de outros problemas envolvendo a cinética de compostos.

- Construção de um banco de dados com parâmetros de interesse para os modelos compartimentais apresentados nas publicações mais recentes da ICRP, incluindo os modelos biocinéticos específicos dos radionuclídeos de interesse do IPEN. 


\section{DOSIMETRIA}

\subsection{Evolução dos Modelos Biocinéticos}

Um modelo biocinético é uma descrição matemática da distribuição e da eliminação de uma substância no corpo, em função do tempo, após sua incorporação. A estimativa das doses nos órgãos, resultante da deposição dos radionuclídeos nestes, é feita com base nos modelos recomendados nas publicações da ICRP.

Um radionuclídeo pode se distribuir pelo corpo inteiro ou ficar concentrado em um tecido, ou um órgão específico, como é o caso da tireóide para o Iodo, pois o organismo não distingue o isótopo I-131 do iodo natural. Assim, elementos de uma mesma família química apresentarão distribuição sistêmica semelhante (ICRP-78, 1997).

Na primeira publicação da ICRP, em 1959, havia pouca informação disponível sobre a cinética dos radionuclídeos no corpo humano. Os modelos foram elaborados com base em dados obtidos em experiências com animais, ou quando possível, a partir de dados experimentais de indivíduos expostos à radionuclídeos. Os modelos apresentavam diversas simplificações, pois os cálculos seriam realizados manualmente (Lourenço, 2007).

As publicações subseqüentes da ICRP, das décadas de 1960 e 1970 apresentaram uma variada coletânea de dados fisiológicos, anatômicos e bioquímicos obtidos a partir de estudos mais elaborados, culminando na publicação 30 de 1979, que considera a recirculação de material entre os órgãos. Apesar dos modelos apresentarem resoluções matemáticas mais complexas, computadores de grande porte e calculadoras programáveis já estavam disponíveis no mercado.

Segundo Sanches (2004), os avanços na área da dosimetria interna fizeram com que a ICRP introduzisse modificações importantes nos modelos biocinéticos apresentados na sua publicação 30 (ICRP, 1979). As publicações 54 (ICRP, 1988), publicações 56 (ICRP, 1990), 67 (ICRP, 1994) e 69 (ICRP, 1995), da ICRP revisaram os modelos biocinéticos para alguns radionuclídeos. Em 1994 a publicação 66 (ICRP, 1994) apresentou um novo modelo biocinético para o trato respiratório. 
Os coeficientes de dose para trabalhadores são apresentados na publicação 68 (ICRP, 1994), juntamente com os de dose efetiva comprometida por unidade de incorporação, esta publicação também introduz o novo modelo para o trato respiratório e modelos sistêmicos atualizados.

Estas ações realizadas pela ICRP culminaram na publicação de um novo guia para o projeto de um programa de monitoração interna e interpretação dos resultados, para um grupo de radionuclídeos importantes na exposição ocupacional. (ICRP 78, 1997).

As normas nacionais vigentes (CNEN, 2005) seguem as recomendações e os modelos dosimétricos baseados na publicação ICRP 66 (1991), porém o presente trabalho irá utilizar os dados apresentados na publicação ICRP 78 como parâmetros para validação de sua eficácia.

Em 2007 a ICRP 100 (2007) forneceu um novo modelo para o trato alimentar humano (HRTM - Human Alimentary Tract Model), que, em conjunto com o modelo respiratório da publicação 66, substitui definitivamente os modelos publicados na publicação 30, de 1979. Este novo modelo leva em consideração importantes diferenças entre homens e mulheres, crianças e adultos. A publicação 30 inicia o modelo do trato alimentar pelo compartimento que representa o estômago e o único ponto de transferência para o sangue era o intestino delgado. No novo modelo apresentado, isto permanece igual para a maioria dos radionuclídeos, mas também considera a possibilidade de retenção e absorção para o sangue em todas as regiões, exceto o esôfago.

Atualmente dois grupos de trabalho da ICRP estão concentrados na revisão dos modelos biocinéticos, onde serão recalculados os coeficientes de dose para incorporação de radionuclídeos. Estes dados substituirão os fornecidos pelas publicações 30, 68, 54 e 78 (INDOS, 2011).

Apesar deste trabalho não utilizar o novo modelo do trato alimentar humano publicado, ele foi concebido com o requisito fundamental de adaptar-se a futuras mudanças nos modelos publicados, permitindo alterações nos modelos gravados de forma rápida e fácil. Assim, quaisquer mudanças nos modelos a serem publicados pela ICRP poderão ser 
atualizadas pelo programa proposto, bastando que o usuário informe os novos compartimentos e seus respectivos coeficientes de transferência.

\subsection{Metodologia da ICRP para Avaliação da Dose}

Segundo as recomendações da ICRP para a estimativa da atividade incorporada ou incorporação I, quando uma única medida experimental $\mathrm{M}$ é realizada, é determinada por:

$$
I=\frac{M}{m(t)}
$$

onde: $m(t)$ é a fração da incorporação unitária em um compartimento no tempo t após a incorporação, calculada pelos modelos compartimentais.

Multiplica-se esta incorporação I pelo coeficiente de dose para se obter a dose efetiva, que é comparada com os níveis de limite de dose para o caso específico. Se for notada uma dose que excede os limites recomendados, é realizada uma investigação mais detalhada, onde são feitas outras medidas, que confirmem ou refinem a estimativa inicial.

A dose efetiva comprometida, E(50), é obtida pelo produto da incorporação, I, pelo coeficiente de dose, e(g), para o radionuclídeo em questão é expresso pela equação (2):

$$
E=I . e(g)_{j}
$$

Em uma monitoração de rotina, o dia exato da incorporação pode ser desconhecido. Segundo a ICRP, assume-se que a incorporação ocorreu no meio do intervalo da monitoração T:

$$
I=\frac{M}{m(T / 2)}
$$


2.3 Revisão dos métodos e códigos computacionais disponíveis

Vários códigos computacionais foram desenvolvidos com o objetivo de determinar doses de incorporações, por diferentes grupos de pesquisadores ao redor do mundo. O trabalho de Ansoborlo et al. (2003) faz uma comparação entre seis destes programas de computador, seguindo critérios definidos pela ICRP.

Os programas foram avaliados em quesitos técnicos:

- facilidade de instalação

- geração de gráficos

- possibilidade de gravar os resultados

- facilidade de manuseio da aplicação

- ajuda ao usuário

e critérios mais importantes referentes ao seu uso no cálculo de doses:

- quantidade de elementos disponíveis

- formas de incorporação previstas

- variedades nos parâmetros de incorporação

- pontos de medida, quantidade de órgãos ou excretas

entre outros.

Entre os programas avaliados por Ansoborlo, está o MONDAL (monitoring dose assessment), criado pelo Instituto de Ciências Radiológicas Nacional (NIRS) do Japão, que posteriormente foi atualizado para uma segunda versão, o MONDAL 2, atualizado com as então recentes alterações do modelo do trato respiratório humano da ICRP (Ishigure, 2004).

Outro programa avaliado foi o IMBA (integrated modules for bioassay analysis), desenvolvido por Birchall (2007), este programa evoluiu para outras versões nos anos seguintes ao seu desenvolvimento: Expert, Professional e Professional Plus. Sua principal característica é possuir parte de suas funcionalidades separadas em módulos que podem ser adicionadas conforme a necessidade. 
A gerência de radioproteção do IPEN faz uso do AIDE (Activity and Internal Dose Estimates), desenvolvido por Bertelli et al. (2008) com a participação de pesquisadores do Instituto de Radioproteção e Dosimetria da CNEN (Comissão Nacional de Energia Nuclear) no Rio de Janeiro.

O AIDE também divide suas funcionalidades em módulos, e um deles permite a edição de modelos compartimentais, porém sem apresentar uma representação gráfica para a visualização do modelo.

Dois códigos computacionais importantes avaliados durante o desenvolvimento deste trabalho foram:

- LUDEP, (Lung Dose Evalution Program) desenvolvido por Jarvis e Birchall (1994), um dos primeiros a implementar as alterações do trato respiratório proposto na ICRP 66.

- Humorap (Sanchez, 2003), elaborado como um pacote do software Mathematica, faz uso das capacidades simbólicas deste para resolver analiticamente os modelos biocinéticos.

2.4 Programa de monitoração individual interna do IPEN

A monitoração pode, como uma das formas de proteção radiológica, ter um caráter preventivo quando utilizada para alertar o trabalhador sobre a presença de radiação em uma área, ou confirmatório, avaliando a dose recebida pelo indivíduo, neste caso chamado de monitoração individual.

O IPEN possui instalações onde ocorre o manuseio de materiais radioativos sob a forma não selada, o que aumenta o risco de contaminação. Apesar de toda proteção radiológica envolvida, falhas humanas ou nos dispositivos de segurança envolvidos podem acarretar em uma contaminação.

A contaminação pode ser externa, no caso em que o material entra em contato com a pele ou com as roupas do indivíduo, ou interna, quando o material for incorporado. Esta incorporação pode ocorrer pela inalação ou ingestão da substância ou em casos mais raros, através de um ferimento na pele. 
Segundo a ICRP 75 (1997), programas de monitoração são divididos em:

- monitoração rotineira, associada a uma operação contínua, demonstra se os níveis de dose individuais se mantêm satisfatórios.

- monitoração relacionada à tarefa, que se aplica a uma operação específica. Fornece dados para tomada de decisão no gerenciamento da operação e dá suporte à otimização da proteção.

- monitoração especial, que possui uma natureza investigativa, e fornece informações detalhadas sobre uma situação de forma a elucidar problemas e definir procedimentos a serem adotados.

O diagrama da FIG. 1 representa o programa de monitoração interna do IPEN, na qual cada instalação radiativa possui um supervisor responsável pelo programa de proteção radiológica. Este supervisor encaminha os trabalhadores, ou as amostras, para medidas na Gerência de Metrologia das Radiações (GMR), com uma frequência que pode variar de acordo com o programa de monitoração adotado para aquele radionuclídeo.

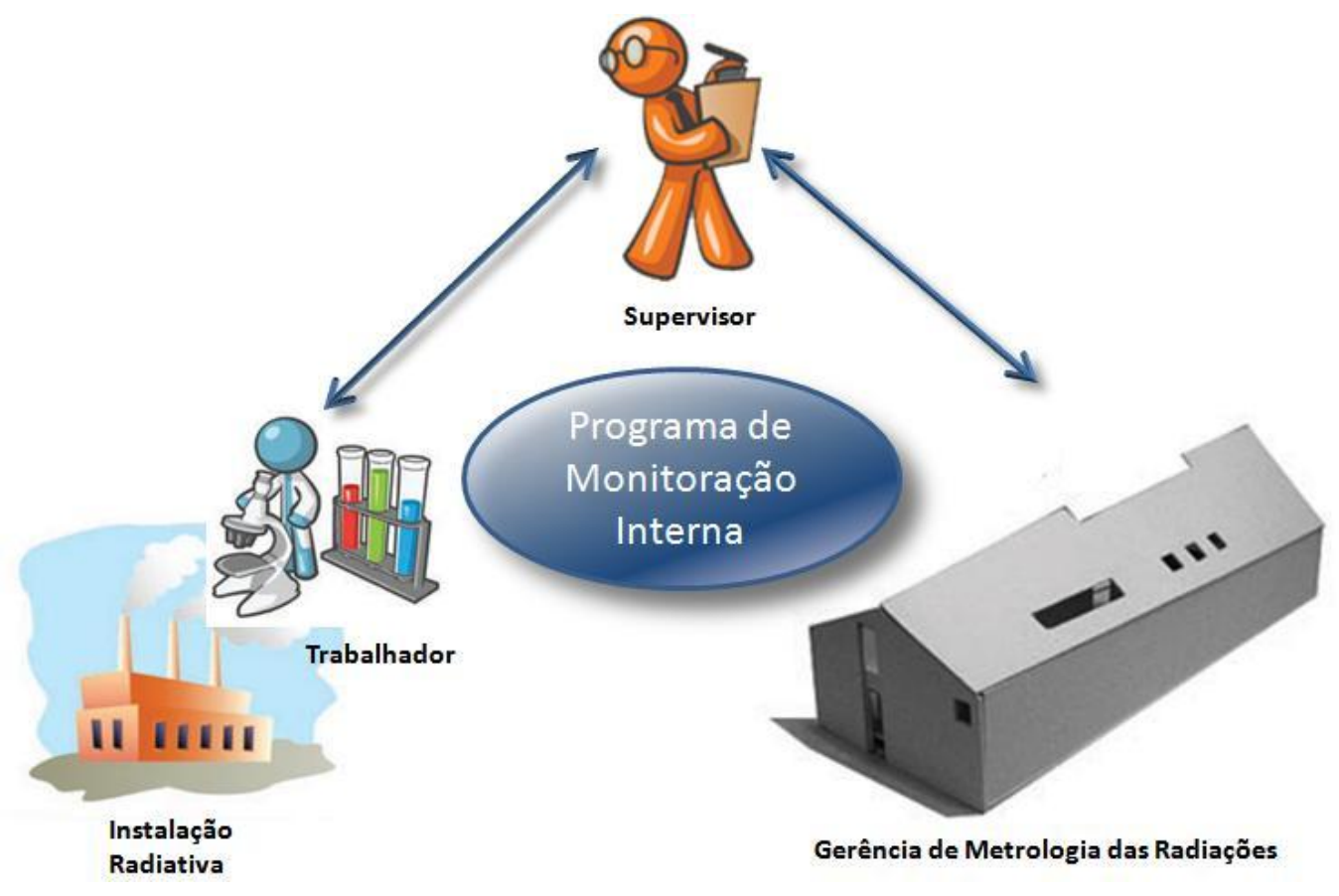

FIGURA 1 - Diagrama do programa de monitoração interna

A GMR realiza a monitoração individual por duas técnicas de medida de atividade: in vivo e in vitro. 
A medida in vivo consiste basicamente na utilização de contadores de corpo inteiro, que são detectores de radiação acoplados a equipamentos de contagem de radiação, permitindo a estimativa da atividade existente no corpo ou em um órgão.

A medida in vitro consiste na avaliação da quantidade de material radioativo presente no corpo, por meio da bioanálise de excretas do indivíduo. Este método é aplicável a todos radionuclídeos, inclusive os emissores de baixas energias. Porém envolve técnicas mais complexas tanto na separação química do elemento radioativo, quanto no uso de um modelo matemático preciso que relaciona, em função do tempo, a quantidade de material presente nas excretas com a quantidade incorporada, ou retida nos órgãos do corpo. 


\section{MODELOS COMPARTIMENTAIS}

Em um modelo biocinético representado como um modelo compartimental, onde cada órgão ou região do organismo é um compartimento, é possível simular o comportamento de um radionuclídeo no corpo humano, e sua distribuição pelos órgãos em função do tempo, seja em horas, dias, ou anos. Os modelos compartimentais também são muito utilizados nos estudos farmacocinéticos (Rescigno, 2004).

Segundo Sanchez (2003), um modelo compartimental é uma rede onde os "nós" são compartimentos conectados por "flechas", representando o fluxo de uma substância de um compartimento para outro. Diversos métodos matemáticos têm sido utilizados para resolver estes modelos, onde o mais comum é representá-los como um sistema de equações diferenciais. Conforme aumenta o número de compartimentos e suas conexões, maior é o desafio para encontrar as soluções algébricas e numéricas para o modelo.

3.1 Modelos para a representação da incorporação, transferência e liberação do material.

A publicação 78 da ICRP consolida modelos que descrevem a cinética dos radionuclídeos considerados importantes na exposição ocupacional. Estes modelos são subdivididos em três partes principais: modelo para o sistema respiratório, modelo para o sistema gastrointestinal e modelos sistêmicos específicos descrevendo os processos de distribuição, retenção e eliminação para cada radionuclídeo.

\subsubsection{Modelo dosimétrico para o sistema respiratório}

Um modelo específico para absorção do material por vias aéreas é apresentado separadamente do modelo sistêmico de cada radionuclídeo. As taxas de transferência entre os compartimentos deste modelo não variam em função do radionuclídeo em questão, mas sim para cada tipo de composto em que o radionuclídeo está presente.

O modelo do trato respiratório descrito na ICRP 66 é representado por 5 regiões: nasal anterior, laringe e faringe, bronquial, bronquiolar e alveolar intersticial (FIG. 2). 


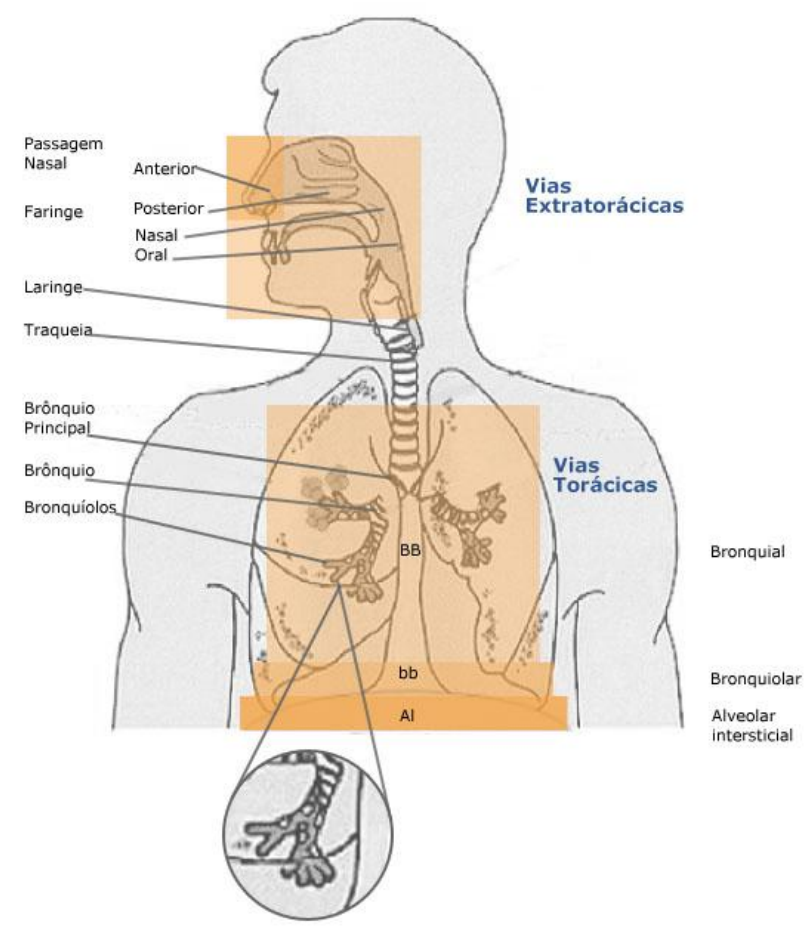

FIGURA 2 - Regiões do sistema respiratório humano. (Adaptado da ICRP 66)

Cada uma destas cinco regiões contém compartimentos e apenas estes são considerados na modelagem resolvida matematicamente. Estes compartimentos são os "pontos de entrada" do sistema, no caso de uma incorporação por inalação do material (FIG 3).

O compartimento da região nasal anterior, denominado ET1 não é considerado na modelagem, pois o material depositado nesta região retorna para o ambiente sem ser absorvido pelo organismo. 


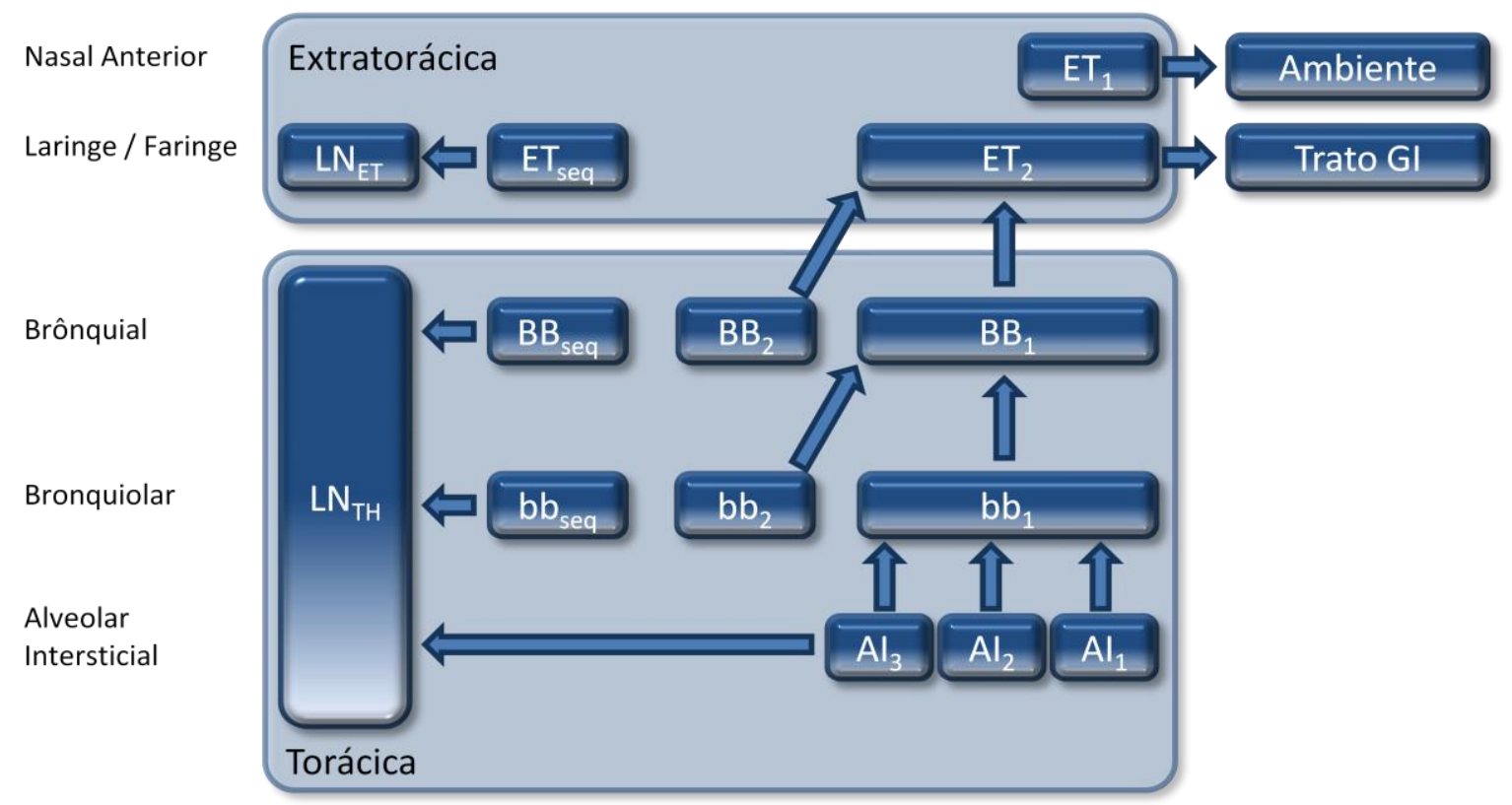

FIGURA 3 - Modelo compartimental descrevendo o transporte de partículas em cada região do trato respiratório. (Adaptado da ICRP 78)

Este modelo é muito mais complicado que seus predecessores, e foi projetado para representar, tão realisticamente quanto possível, a deposição e liberação de partículas em cada região do trato respiratório. (Frappe e Rannou, 1998).

A deposição de um aerossol nas diversas regiões do sistema respiratório varia conforme algumas características aerodinâmicas das partículas, como o Activity Median Aerodynamic Diameter - AMAD (ou DAMA, Diâmetro Aerodinâmico Mediano em Atividade). Partículas de AMAD menor podem se depositar em maiores quantidades nas vias aéreas inferiores, como os alvéolos pulmonares (ICRP 78, 1997).

A publicação 30 da ICRP utilizava como padrão um AMAD de $1 \mu \mathrm{m}$, e a partir da publicação 68 este valor padrão recomendado passou a ser de $5 \mu \mathrm{m}$ para trabalhadores ocupacionais.

A ICRP 66 apresenta as frações de deposição para cada compartimento, relativo às partículas do $\mathrm{AMAD}$ utilizado. $\mathrm{Na}$ TAB. 1 são apresentados os valores de entrada do modelo compartimental. 
TABELA 1 - Partição da deposição $(A M A D=5 \mu \mathrm{m})$ nas regiões do trato respiratório e seus compartimentos, de acordo com o modelo da ICRP 66.

\begin{tabular}{|c|c|c|c|c|}
\hline Região & \% Depositado & Compartimento & Fração & Total \\
\hline \multirow{2}{*}{ ET2 } & \multirow{2}{*}{40} & ET2 & 0,9995 & 0,3998 \\
\hline & & ETseq & 0,0005 & 0,0002 \\
\hline \multirow{3}{*}{ BB } & \multirow{3}{*}{1.8} & BB1 & 0,66 & 0,011880 \\
\hline & & BB2 & 0,333 & 0,005994 \\
\hline & & BBseq & 0,007 & 0,000126 \\
\hline \multirow{3}{*}{$\mathrm{bb}$} & \multirow{3}{*}{1.1} & bb1 & 0,596 & 0,006556 \\
\hline & & bb2 & 0,397 & 0,004367 \\
\hline & & bbseq & 0,007 & 0,000077 \\
\hline \multirow{3}{*}{ Al } & \multirow{3}{*}{5.3} & Al1 & 0,3 & 0,0159 \\
\hline & & $\mathrm{Al} 2$ & 0,6 & 0,0318 \\
\hline & & $\mathrm{Al} 3$ & 0,1 & 0,0053 \\
\hline
\end{tabular}

A coluna Total é obtida pela multiplicação da coluna com o percentual depositado na região, pela coluna com a fração específica de um determinado compartimento.

É importante observar que a soma dos percentuais não é $100 \%$ porque parte do material foi depositado em ET1, e não é considerado nos valores relacionados.

Após o processo de deposição, os materiais se distribuem pelo sangue e pelo sistema gastrointestinal em um processo de liberação, onde os compostos são divididos em três classes de inalação, conforme sua permanência na região pulmonar: $\mathrm{F}$ (Fast), M (Moderate) e S (Slow). Esta classificação substitui respectivamente as classes D, W e Y, que eram utilizadas na publicação 30 da ICRP.

Em compostos do tipo S, o processo de absorção do material inalado acontece de forma lenta. Os valores mostrados na FIG. 4 demonstram que nesta categoria de particulado, $0,1 \%$ do material depositado em cada região é absorvido com uma meia-vida biológica de 10 minutos, e 99,9\% com uma meia-vida de 7000 dias.

Em compostos do tipo $\mathrm{M}, 10 \%$ são absorvidos com uma meia-vida de 10 minutos, e $90 \%$ com uma meia-vida de 140 dias, e para o tipo F, 100\% do material é absorvido com meia-vida de 10 minutos ocasionando uma rápida absorção de quase todo material depositado nos brônquios e nos alvéolos pulmonares. 
Podemos considerar que cada compartimento do sistema respiratório que recebe o material inalado em seu estado inicial possui um compartimento espelho representando o material em estado transformado. Este modelo é comum para qualquer elemento, e as taxas de dissolução estão relacionadas com a forma química do elemento. (Sanchez, 2003).

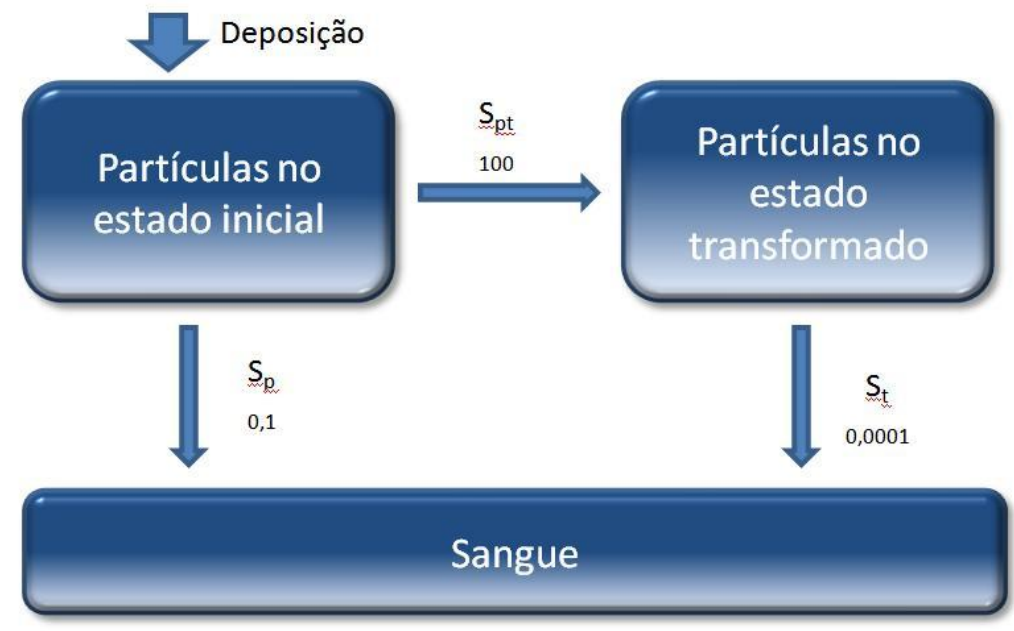

FIGURA 4 - Representação da dissolução de um composto do tipo $S$ em função do tempo no trato respiratório

Assim, em todos os casos de incorporação de materiais nas classes S e M, os compartimentos do modelo respiratório serão duplicados, de forma que o outro represente o mesmo compartimento, com as partículas em estado transformado. Para compostos do tipo $\mathrm{F}$, este procedimento não é necessário, uma vez que quase todo material depositado é absorvido diretamente pelo sangue rapidamente.

\subsubsection{Modelo dosimétrico para o sistema gastrointestinal}

O material pode chegar ao sistema gastrointestinal diretamente pela ingestão, ou indiretamente, transferido pelo trato respiratório. A FIG. 5 apresenta o modelo proposto pela ICRP 30, que posteriormente foi revisado pela ICRP 100. 


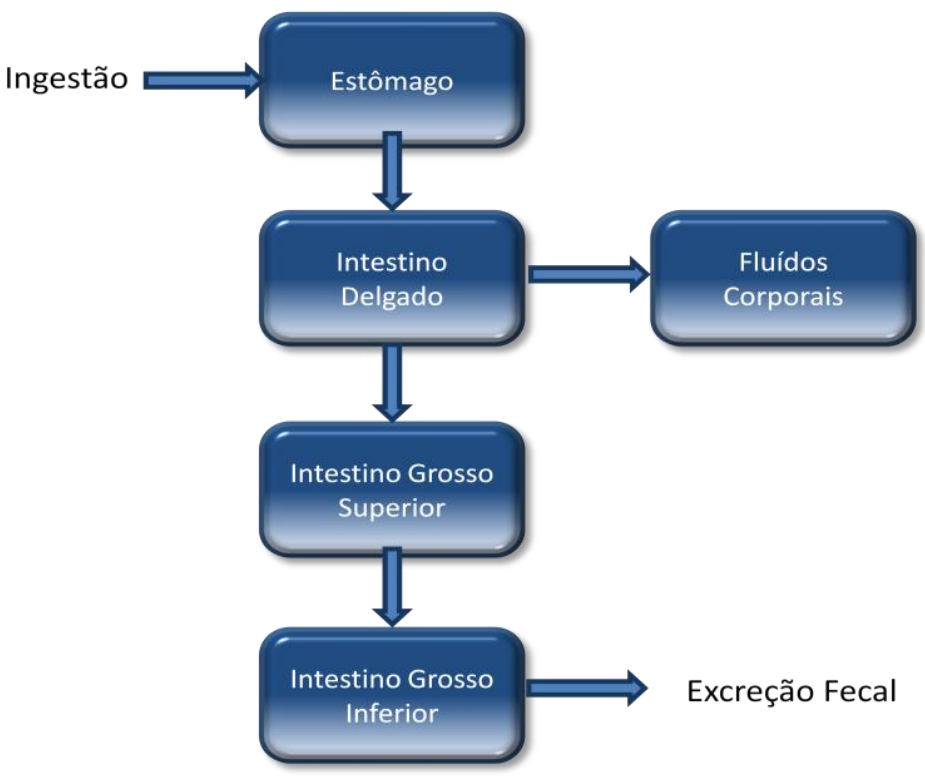

FIGURA 5 - Modelo matemático que descreve a cinética dos radionuclídeos no trato gastrointestinal apresentado pela ICRP 30

O trato gastrointestinal é representado por 4 seções, cada uma é considerada um único compartimento e a transferência do material ocorre em um único sentido, sem realimentação dos compartimentos.

Os valores das constantes de transferência do trato gastrointestinal são as mesmas para todos os radionuclídeos com exceção apenas para a transferência do intestino delgado para o sangue, que é determinada pela equação 4.

$$
\lambda_{B}=\frac{\left(f 1 \cdot \lambda_{S I}\right)}{(1-f 1)}
$$

Onde $\lambda_{S I}$ é a constante de transferência do intestino delgado para o intestino grosso, e $f 1$ é fornecido pela ICRP e varia conforme o composto onde o elemento está presente.

A publicação 100 da ICRP de 2007 fornece um novo modelo do trato alimentar humano (HATM - Human alimentary tract model) que complementa o modelo do trato respiratório humano apresentado na publicação 66. Juntos, estes dois modelos substituem os modelos originalmente apresentados publicados na ICRP 30. 
Enquanto o modelo da publicação 30 inicia o sistema gastrointestinal pelo estômago e a absorção de material para o sangue apenas pelo intestino delgado, este apresentado pela ICRP 100 (FIG. 6), considerando dados de estudos realizados desde 1979, considera a possibilidade de retenção e absorção de material em todas as regiões, exceto o esôfago.

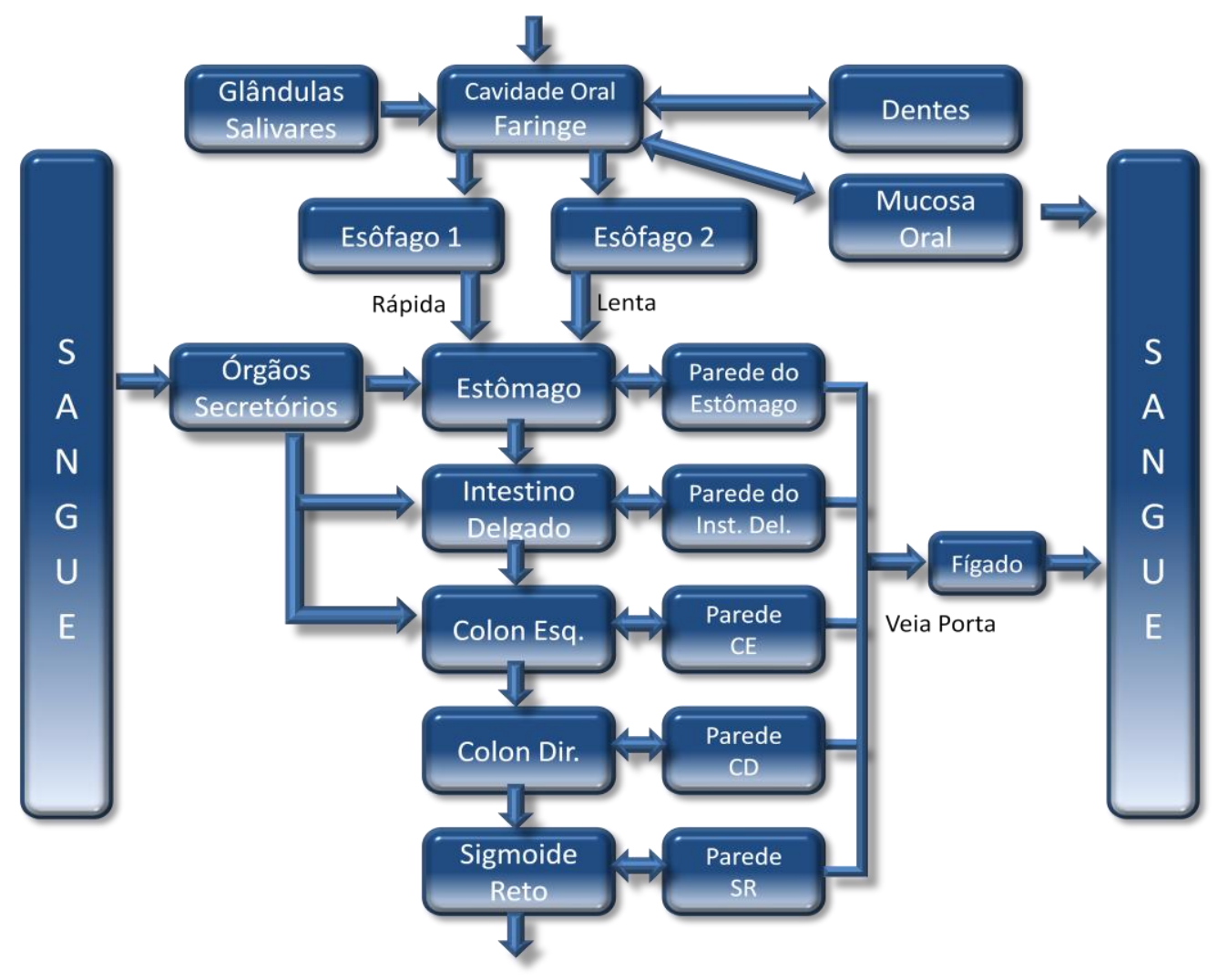

FIGURA 6 - Modelo do Trato Alimentar Humano apresentado pela ICRP 100

Este modelo apresenta um maior refinamento na fisiologia do sistema humano do que o anterior, compreendendo desde a boca, esôfago, estômago, intestino grosso e delgado, o reto e o canal anal. Ele fornece a flexibilidade necessária para calcular doses para uma extensa variedade de condições de exposição e para indivíduos específicos, por considerar diferenças de idade e sexo (Stather, 2004).

\subsection{Descrição matemática dos modelos compartimentais}

Segundo Sanchez (2003), um modelo compartimental pode ser representado por um sistema de equações diferenciais de primeira ordem com coeficientes constantes. 
Quando o número de compartimentos é pequeno, e as entradas do sistema seguem uma função variável no tempo, o método mais adequado para resolver o sistema costuma ser o das transformadas de Laplace. Porém, quando o grau de complexidade do sistema aumenta, tendo uma quantidade de compartimentos elevada, e as relações entre eles também se tornam complexas, e neste caso pode-se aconselhar a utilização de métodos numéricos. Normalmente o prático é recorrer a uma combinação de métodos (Rodrigues, 1993).

Em um modelo compartimental onde os compartimentos são representados por números $\mathrm{i}=1,2,3 \ldots \mathrm{n}$, de forma que $\lambda_{i, j}$ seja a taxa de transferência do compartimento $\mathrm{i}$ para o compartimento $\mathrm{j}$, então o valor que representa a taxa total de saídas do compartimento i será

$$
\lambda_{i}=\sum_{j=1, j \neq i}^{n+1} \lambda_{i, j}
$$

Seja $q_{i}(t)$ a retenção no compartimento $i$ no tempo $t$ e $b_{i}(t)$ a entrada no compartimento i no tempo t, então a derivada de $\mathrm{q}_{i}(\mathrm{t})$ representa a variação da retenção no compartimento i no tempo $\mathrm{t}$

$$
\begin{array}{r}
\frac{d q_{1}(t)}{d t}=\sum_{r} \lambda_{r, 1} q_{r}(t)-\sum_{j} \lambda_{1, j} q_{1}(t)+b_{i}(t)= \\
=\sum_{r} \lambda_{r, 1} q_{r}(t)-\lambda_{1} q_{1}(t)+b_{i}(t) \\
\vdots \\
\frac{d q_{n}(t)}{d t}=\sum_{r} \lambda_{r, n} q_{r}(t)-\sum_{j} \lambda_{n, j} q_{n}(t)+b_{i}(t)= \\
=\sum_{r} \lambda_{r, n} q_{n}(t)-\lambda_{n} q_{n}(t)+b_{i}(t)
\end{array}
$$


Assim, a variação da quantidade eliminada no tempo t para o sistema completo é dada pela equação

$$
\frac{d q_{n}(t)}{d t}=\sum_{r} \lambda_{r, n+1} q_{r}(t)
$$

Esta equação diferencial pode ser reescrita de forma matricial como

$$
\frac{d q(t)}{d t}=A \cdot q(t)+b(t)
$$

Onde a matriz A tem valores não-positivos na diagonal e valores não-negativos nos demais elementos

$$
\begin{gathered}
A=\left(\begin{array}{ccccc}
-\lambda_{1} & \lambda_{2,1} & \cdots & \lambda_{n, 1} & 0 \\
\lambda_{1,2} & -\lambda_{2} & \cdots & \lambda_{n, 2} & 0 \\
\vdots & \vdots & \ddots & \vdots & \vdots \\
\lambda_{1, n} & \lambda_{2, n} & \cdots & -\lambda_{n} & 0 \\
\lambda_{1, n+1} & \lambda_{2, n+1} & \cdots & \lambda_{n, n+1} & 0
\end{array}\right) \\
b(t)=\left(\begin{array}{c}
b_{1}(t) \\
b_{2}(t) \\
\vdots \\
b_{n}(t) \\
0(t)
\end{array}\right), \\
q(t)=\left(\begin{array}{c}
q_{1}(t) \\
q_{2}(t) \\
\vdots \\
q_{n}(t) \\
q_{n}+1(t)
\end{array}\right)
\end{gathered}
$$




\section{PROJETO}

O desenvolvimento do programa foi executado de acordo com práticas de engenharia de software, que estabelecem uma metodologia para o ciclo de vida do sistema, passando por fases de visualização, planejamento, construção, e estabilização.

A fase de planejamento, onde são efetuadas a análise e definição dos requisitos consistiu de

- entrevistas com os futuros usuários do programa, responsáveis pela avaliação da dose em casos de incorporação de material do IPEN.

- avaliações dos atuais programas similares disponíveis (Ansorbolo et al. 2003), sejam eles usados no IPEN ou não, como o Ludep, o IMBA (Birchall et al., 2003), e o AIDE (Bertelli et al., 2008).

- estudos sobre a forma como os dados biocinéticos são disponibilizados pelas publicações da ICRP, o que serviu para definir a interface de entrada dos valores dos modelos.

\subsection{Arquitetura do Sistema}

A análise destes requisitos serviu para o estabelecimento do escopo final do projeto, e a definição da arquitetura apresentada na FIG. 7, que demonstra a divisão do programa em dois módulos independentes e o fluxo das informações entre eles.

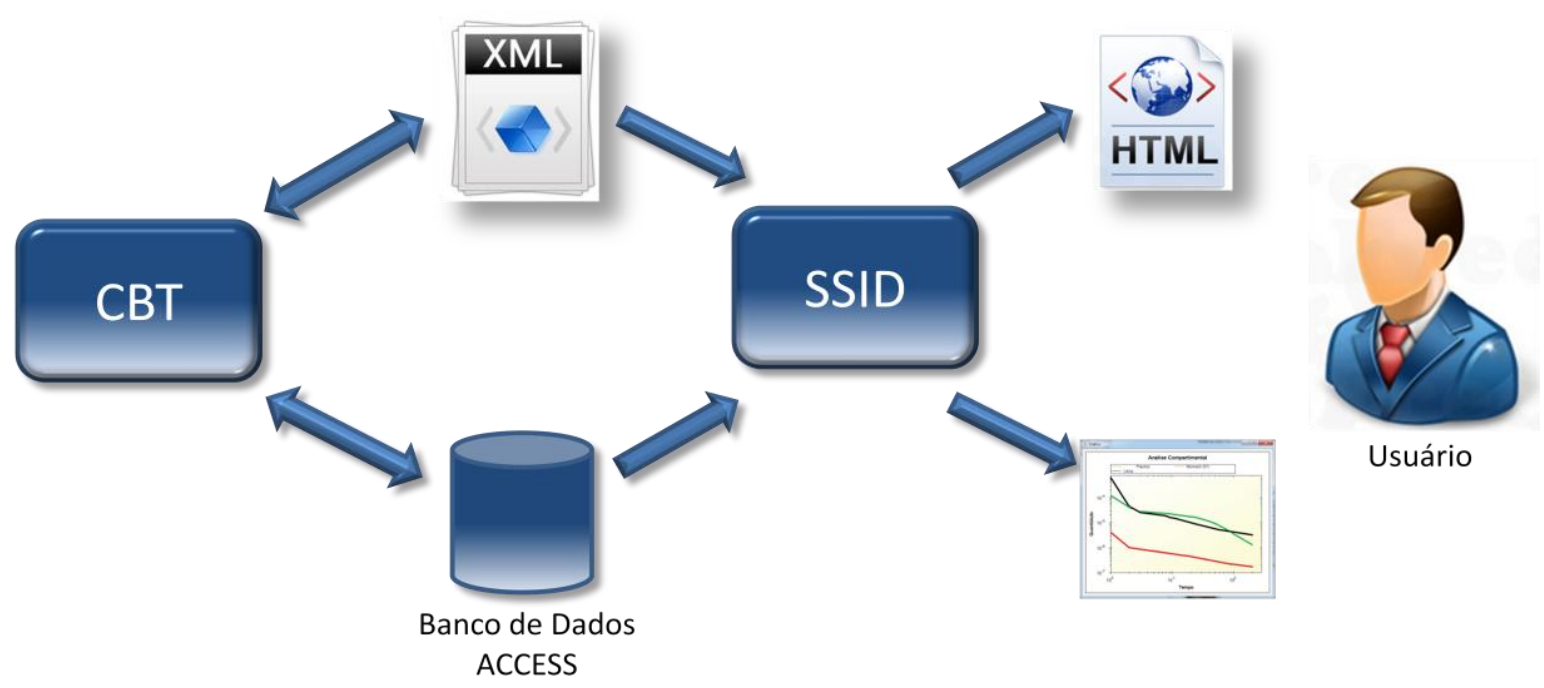

FIGURA 7 - Arquitetura do sistema desenvolvido 
O módulo chamado de CBT (Compartiment Builder Tool - Ferramenta de Construção de Compartimentos) é responsável por gravar e ler informações sobre os modelos, tanto no banco de dados criado no Access quanto em arquivos XML (eXtensible Markup Language), uma linguagem de marcação que define um padrão para troca de informações entre aplicações criadas em diferentes linguagens.

O módulo chamado SSID (Smart Software for Internal Dosimetry - Software Inteligente para Dosimetria Interna) efetua a leitura destes modelos, monta um sistema de equações diferenciais, resolve utilizando um método matemático, e gera como saída uma tabela de dados e um gráfico.

\subsection{Plataforma Computacional}

O programa foi escrito na linguagem de programação C\#, uma linguagem orientada a objetos, lançada pela Microsoft em 2002 para fazer parte das linguagens de programação que compõem sua plataforma de desenvolvimento .NET, hoje o C\# é uma das linguagens de programação mais populares do mercado, ao lado de Java, C, PHP e Visual Basic (Tiobe, 2010).

O Microsoft .NET é uma iniciativa da empresa Microsoft, que visa uma plataforma única para desenvolvimento e execução de sistemas e aplicações. Todo e qualquer código gerado para .NET, pode ser executado em qualquer dispositivo que possua um framework de tal plataforma.

Existem diferentes tipos de aplicações comerciais desenvolvidas no mercado, sendo atualmente dois deles mais comuns, um é chamado de "Web Applications" que depende de um servidor web para funcionar, e são executados através de um aplicativo navegador, como o Internet Explorer, Firefox, Google Chrome, e outros. Outro tipo de aplicativo são os "Windows Forms", programas executáveis que são instalados no computador do usuário final da aplicação, e é exatamente este o tipo de programa desenvolvido nesse trabalho, pois torna o aplicativo independente de um servidor web, o que exigiria uma administração mais complexa. 
Para o armazenamento dos modelos metabólicos, utiliza-se o banco de dados Microsoft Access, que é parte integrante do pacote Office Professional. O Access é recomendado para uso em pequenas quantidades de dados, que não justificam o uso de um banco de dados de grande porte como o Sql Server ou o Oracle, e por isso foi escolhido como local primário de armazenagem dos dados.

Outra forma de armazenar os modelos criados pelo programa é utilizando o padrão XML, que é um formato para a criação de documentos com dados organizados de forma hierárquica, totalmente independente de plataformas de hardware ou software, o que possibilitará que os modelos compartimentais gravados neste formato sejam futuramente lidos por outros aplicativos.

Foi escolhido o formato HTML (HyperText Markup Language) como padrão para apresentação dos valores dos compartimentos após a resolução do mesmo, desta forma permitindo que o usuário do programa salve como um arquivo independente, e possa reabri-lo para posterior visualização em qualquer navegador de internet, sem a necessidade dos programas CBT e SSID. Da mesma forma, o gráfico gerado com as curvas de retenção também pode ser gravado como uma imagem.

\subsection{Compartiment Builder Tool - CBT}

O CBT permite a criação e manipulação dos modelos biocinéticos, e o armazenamento destes para uso posterior.

Para que estes modelos sejam mantidos, utiliza-se um banco de dados criado no programa Microsoft Access, cujo modelo físico é apresentado na FIG. 8. Apesar de o Access permitir o acesso direto aos dados pelo usuário, esta estrutura foi concebida de forma que apenas o programa CBT manipule os dados em sua origem. 


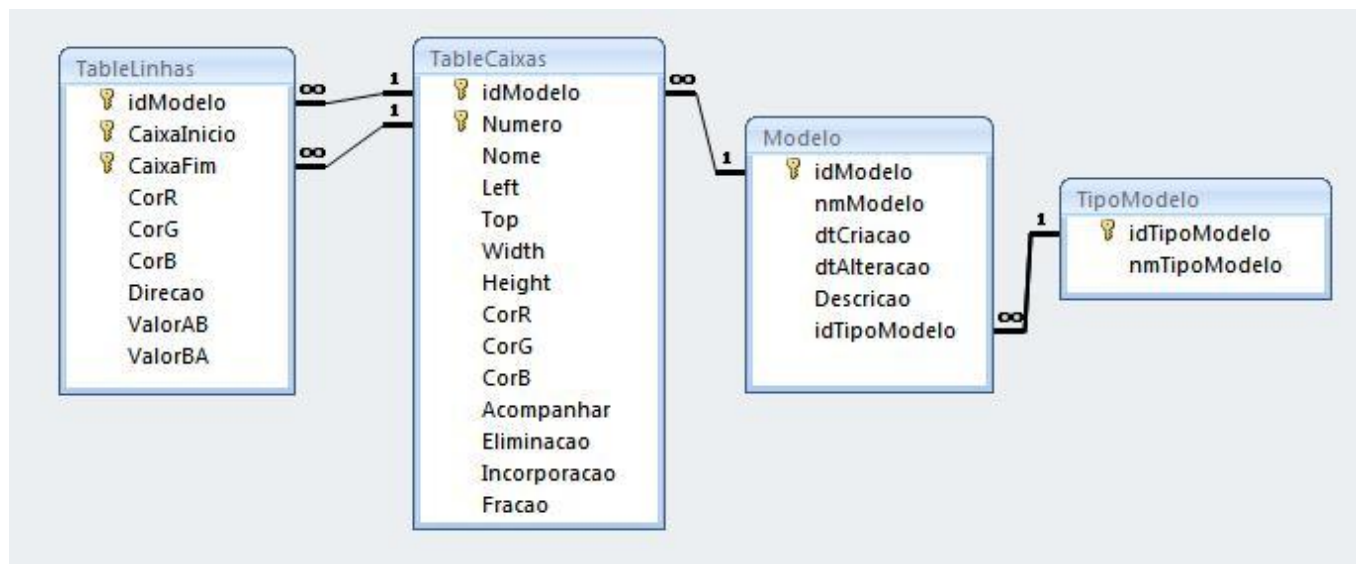

FIGURA 8 - Modelo de banco de dados utilizado

Para iniciar o uso do programa, deve-se optar pela opção "novo" no menu arquivo, para a criação de um modelo, ou abrir um modelo já existente gravado no banco de dados ou em um arquivo XML.

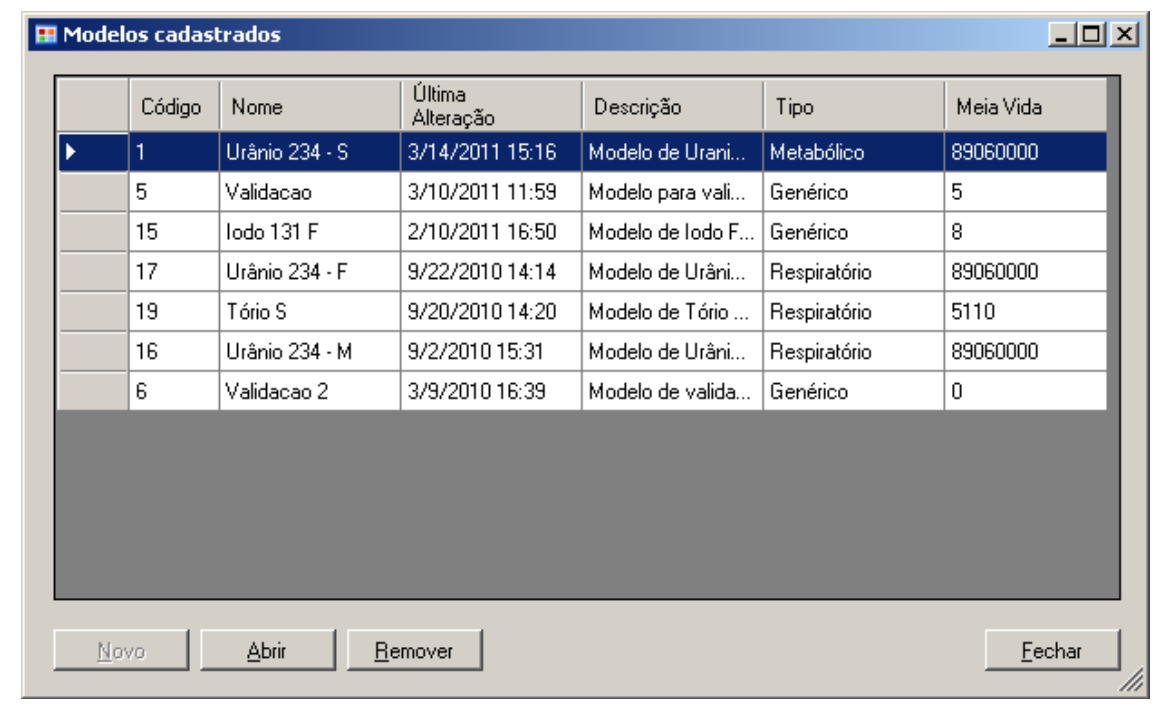

FIGURA 9 - Tela de escolha do modelo a ser utilizado

Na tela exibida na FIG. 9, seleciona-se um modelo clicando na linha desejada, e em seguida no botão “Abrir”. Algumas opções sobre os modelos são exibidas, como seu nome, a data de criação e uma descrição do mesmo.

Com um modelo aberto, o programa apresenta a tela que pode ser observada na FIG. 10 onde todas as funcionalidades do software estão concentradas. Dividida horizontalmente, esta tela apresenta duas áreas bem distintas, a inferior, onde é apresentada uma visualização gráfica do modelo, com os compartimentos representados por retângulos, 
suas respectivas transferências, e as taxas associadas a estes, e a área superior, que permite a inserção e a edição dos dados relativos aos compartimentos e às ligações. É possível mover os compartimentos por essa área, com a função de "arrastar-e-soltar" do mouse, o que é intuitivo a qualquer usuário do Windows.

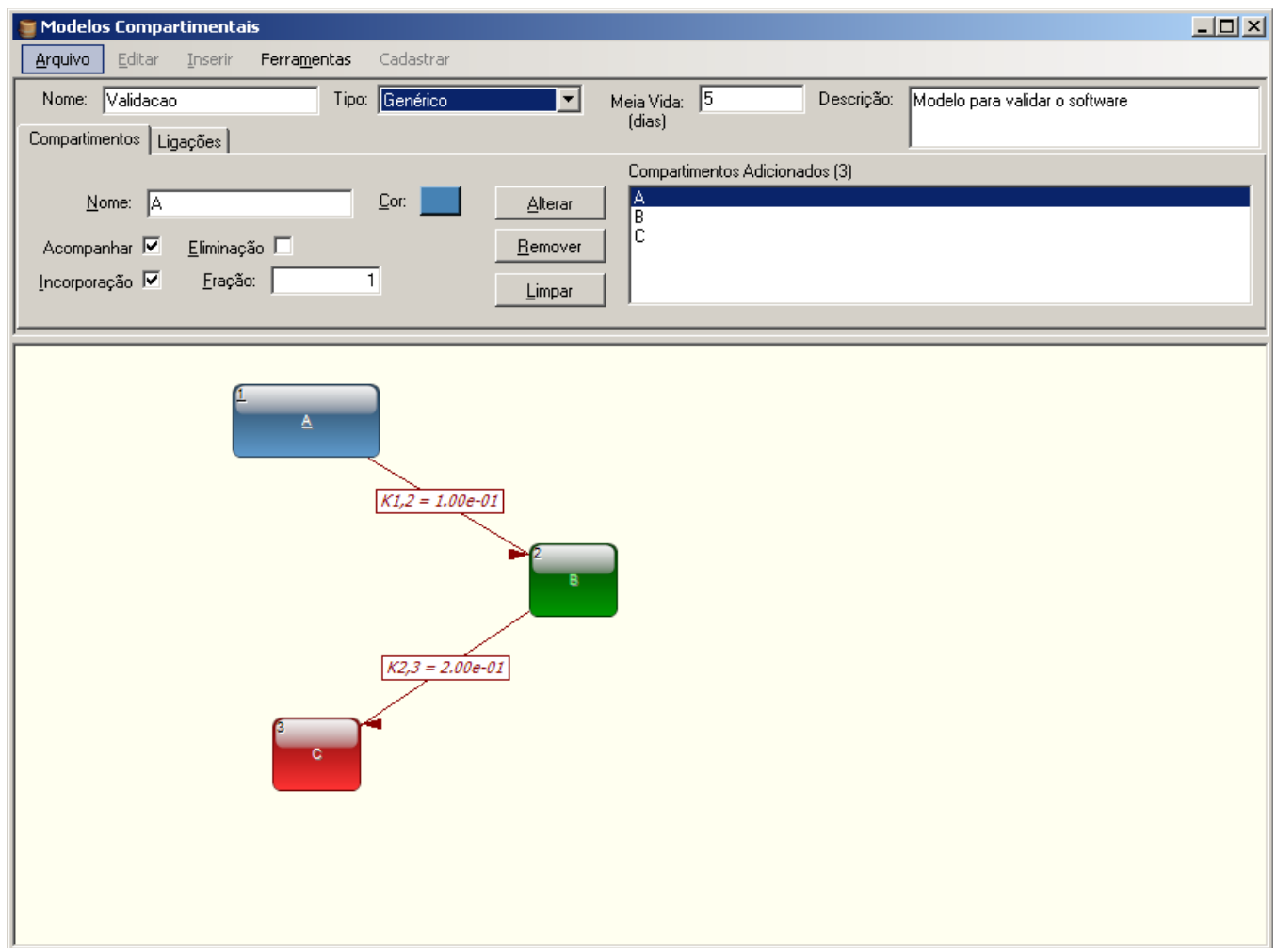

FIGURA 10 - Tela principal do CBT

Em um modelo com muitos compartimentos, o excesso de linhas de ligações pode deixar a visualização um tanto quanto poluída, por isso o software permite, através do menu "ferramentas", que seja desabilitada a exibição das setas, dos rótulos, e até mesmo das linhas de ligações, onde nesse caso, seriam exibidas apenas aquelas do compartimento selecionado. Ainda neste item do menu, pode-se apontar para o arquivo de banco de dados do Access a ser utilizado pela aplicação. Essas informações são todas guardadas em um arquivo de configurações da aplicação, e já estarão configuradas na próxima execução da mesma.

Ao clicar em um compartimento, na região inferior da tela, ou na lista chamada "Compartimentos Adicionados", este passa a ser o compartimento selecionado, sendo possível alterar algumas de suas informações, como seu nome e sua cor. A cor especificada neste momento será a cor da linha exibida no gráfico no outro programa SSID que será 
demonstrado posteriormente. Ainda sobre o compartimento selecionado, a opção “Acompanhar" define que este terá seus valores exibidos, e mostrados como uma linha do gráfico ao resolver o sistema compartimental.

A opção "Eliminação" define este como um compartimento de eliminação, ou seja, a quantidade neste compartimento é apagada a cada tempo t definido ao efetuar o cálculo. Um exemplo comum neste caso seria o compartimento "urina", onde a substância ali depositada é eliminada do sistema.

A opção chamada "Incorporação" faz do compartimento selecionado um ponto de entrada para o sistema compartimental analisado. Uma vez selecionado este compartimento, seu nome será sublinhado na representação gráfica do modelo, para criar uma distinção dos demais, e será solicitado que seja informado o valor da fração de incorporação daquele compartimento. Se a opção incorporação não for habilitada, não é possível especificar esta fração. No caso do corpo humano, em uma incorporação por inalação, diversos compartimentos do sistema respiratório, como faringe, laringe e brônquios serão marcados como "incorporação", e as frações de incorporação específicas de cada um destes são definidas pela ICRP, e calculadas da forma como foi apresentada na TAB. 1 .

A FIG. 11 exibe uma segunda aba nesta mesma tela que permite a criação e modificação das transferências que ocorrem entre os compartimentos, selecionando-os em duas listas, que contém todos adicionados ao modelo até então. Especifica-se então o valor desta transferência, a cor que ela terá na representação visual do modelo. Na lista à direita, todas as transferências existentes são relacionadas.

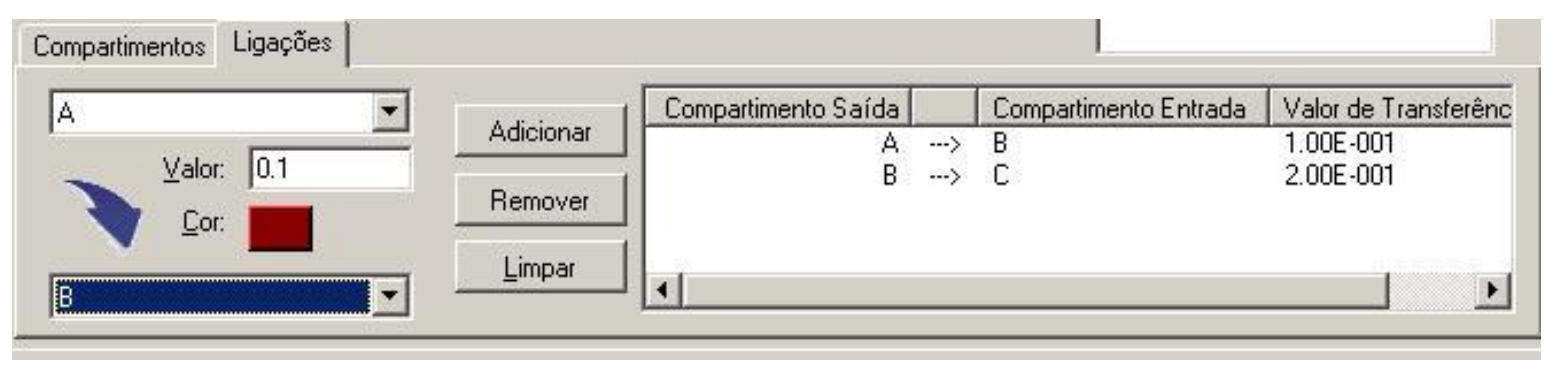

FIGURA 11 - Detalhe da tela do CBT exibindo as ligações 
As transferências possuem uma seta indicando a direção em que ela ocorre, e um rótulo exibindo o valor do coeficiente de transferência entre estes compartimentos. No caso de dois compartimentos que apresentam realimentação dois rótulos são exibidos, bem como duas setas, uma em cada extremidade (FIG. 12).

Apesar de estar graficamente representada com uma única linha, para o modelo compartimental, são duas relações independentes entre os compartimentos, portanto são tratadas como duas ligações diferentes na parte de cima da tela. Elas foram "unidas" na visualização gráfica apenas para fins estéticos, reduzindo a poluição visual no caso de um modelo com muitos compartimentos e ligações.

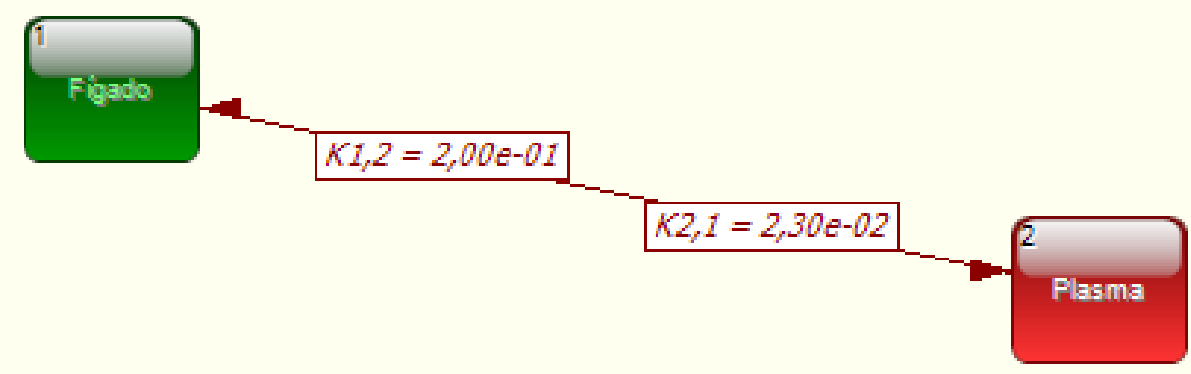

FIGURA 12 - Compartimentos relacionados que apresentam realimentação

Ao concluir a edição do modelo, deve-se gravá-lo, seja sobrescrevendo o atual, ou em um modelo diferente. Deve-se informar o nome do modelo, e a meia vida do radionuclídeo, deixando o valor padrão de zero para elementos que não apresentam decaimento.

Podem-se acrescentar quaisquer outras informações relevantes ao modelo gravado no campo "Descrição", e informar o tipo de modelo que está sendo criado. A informação sobre o tipo do modelo não é utilizada posteriormente na atual versão do SSID, que irá resolver este modelo, porém esse atributo foi concebido a partir da ideia de tornar o CBT flexível de forma que os modelos por ele gerados sejam resolvidos também por outros programas.

No apêndice A é encontrado um exemplo de um arquivo XML gerado pelo CBT, para um modelo simples, com três compartimentos. Este XML foi elaborado de forma a conter a definição de sua estrutura dentro do próprio arquivo, assim, com base 
neste exemplo, podem ser desenvolvidos outros códigos computacionais que façam uso desta estrutura, lendo o arquivo e gerado pelo CBT e aplicando seus próprios métodos de resolução.

\subsection{Smart Software for Internal Dosimetry - SSID}

O SSID lê os modelos compartimentais produzidos pelo CBT e os resolve utilizando um dos métodos matemáticos definidos, apresentando os valores dos compartimentos que foram marcados como "Acompanhar". Assim, a primeira coisa a ser feita, é indicar ao programa a origem dos dados, seja em um arquivo do Microsoft Access, seja um arquivo do padrão XML, que contenha os modelos compartimentais a serem resolvidos.

Ao carregar o modelo, são exibidas as informações que foram cadastradas no CBT: nome do modelo, a descrição, e a meia vida do radionuclídeo, permitindo ao usuário certificar-se que o modelo correto foi carregado.

$\mathrm{Na}$ versão atual do programa, quatro métodos de solução para os sistemas de equações diferenciais são oferecidos, o que torna possível avaliar a precisão e o tempo de processamento de cada um destes métodos. Porém é importante observar que estes fatores dependem não somente do método numérico utilizado, mas também da implementação deste em um algoritmo.

O SSID apresenta uma nova versão da implementação proposta por Rodrigues (1993) para o método semi-analítico criado por Birchall (1989) e três métodos numéricos de resolução de sistemas de equações diferenciais apresentados na biblioteca obtida no DotNumerics (2010).

A FIG. 13 mostra a tela do SSID ao fim do processamento, com o modelo já resolvido. Os resultados são exibidos na forma de tabela. As colunas representam a fração do material incorporado retida no compartimento no instante $\mathrm{t}$, e nos casos de um compartimento de eliminação, valor exibido é o acumulado no intervalo de tempo t-(t-1). 


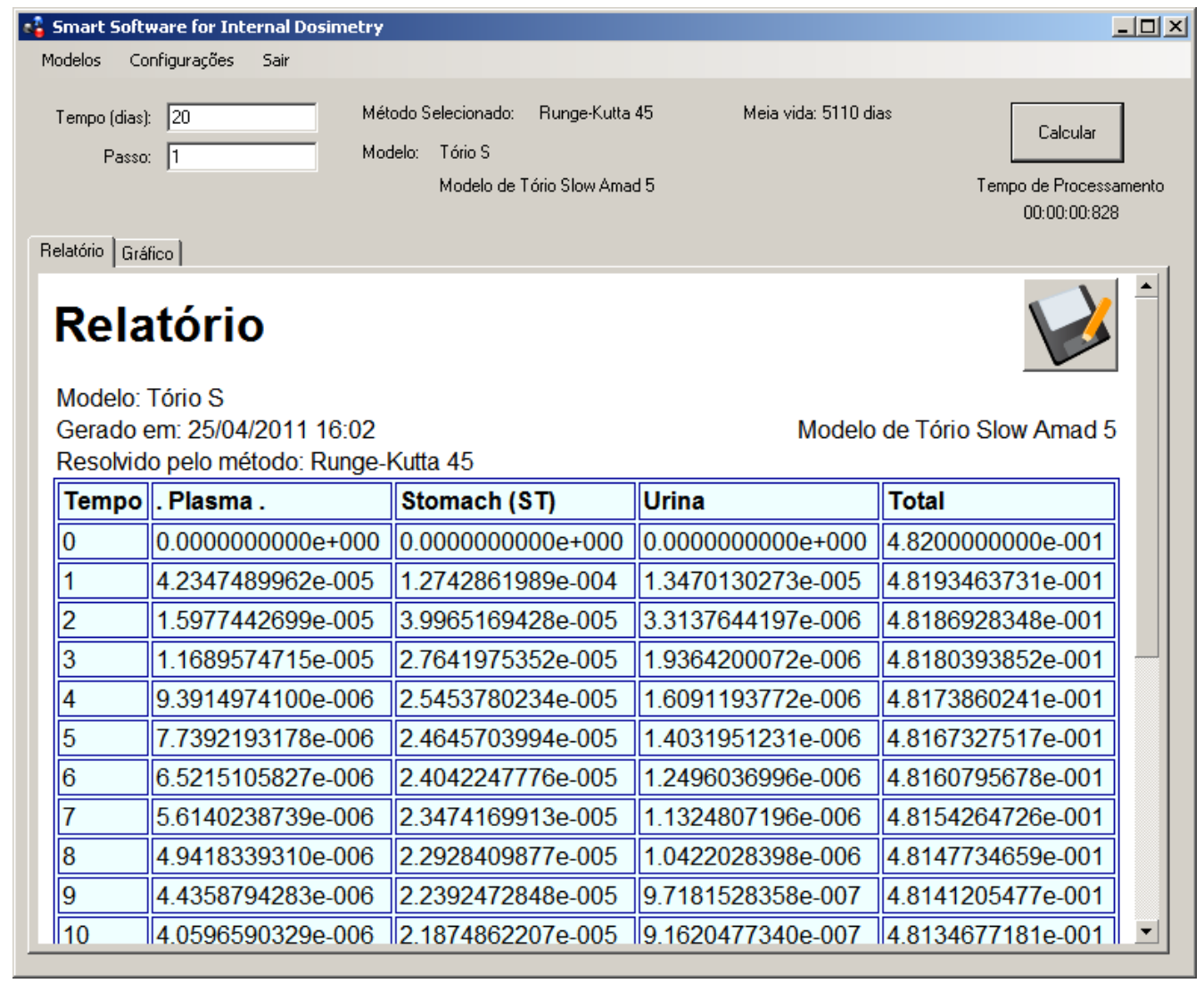

FIGURA 13 - Tela principal do SSID após a resolução de um modelo

Uma última coluna exibe o total de todos os compartimentos (não só dos monitorados). Desta forma, se não houver decaimento radioativo no sistema, o total deve permanecer constante, sempre igual à soma das frações de incorporação informada nos compartimentos de entrada no CBT.

É possível salvar este resultado como um arquivo HTML, o que permite futuras consultas aos dados sem que seja necessário executar o cálculo novamente. $\mathrm{O}$ arquivo HTML pode, como uma página da WEB, ser aberto em qualquer navegador de internet como o Internet Explorer, o Firefox ou o Chrome. Um exemplo deste relatório é apresentado no apêndice B.

Os dados da tabela são apresentados em um gráfico (FIG. 14) onde cada curva corresponde a um compartimento marcado como "acompanhar" na elaboração do modelo no programa CBT. O eixo horizontal é formado em função do tempo informado para o cálculo, e o vertical representa a fração da quantidade contida no dado compartimento. 


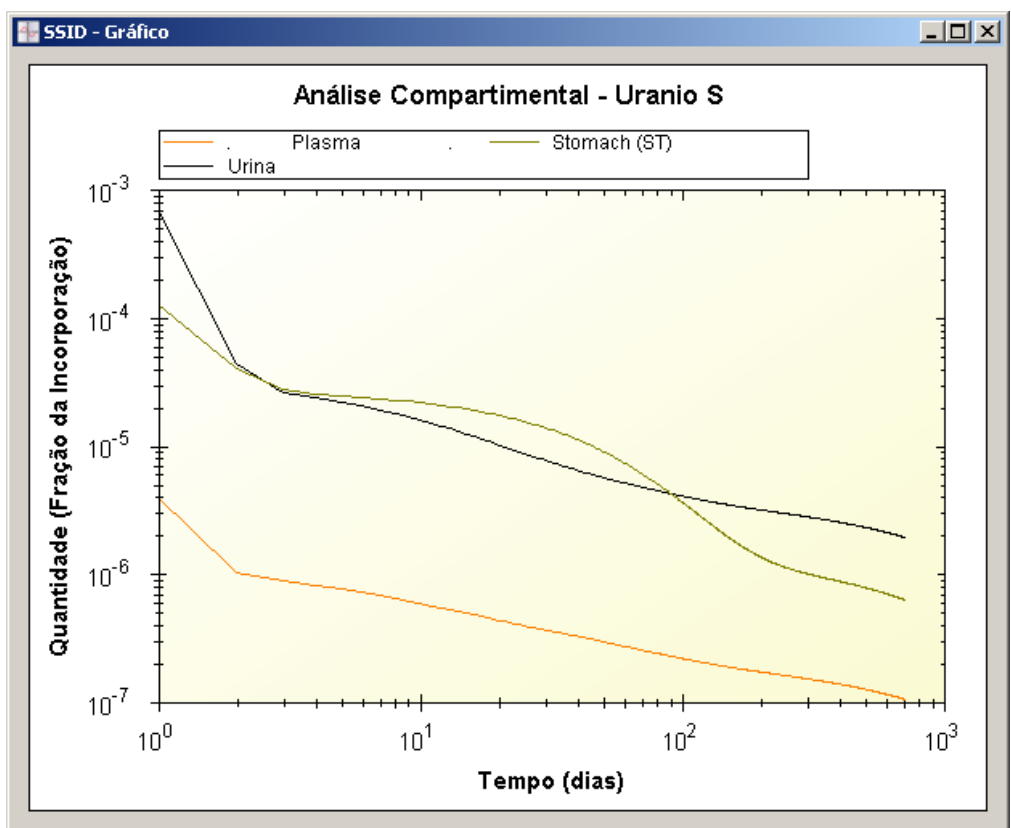

FIGURA 14 - Gráfico gerado pelo SSID após a resolução de um modelo

Uma das configurações desse programa permite que o gráfico seja criado em uma janela a parte, o que torna possível que vários gráficos sejam gerados a partir da resolução do modelo com diferentes variáveis. 


\section{RESULTADOS E DISCUSSÕES}

A primeira validação do programa foi feita com modelos simples, de dois ou três compartimentos como os mostrados até então, e os resultados foram comparados com a solução analítica, nos casos mais simples, ou usando outras aplicações científicas, como o software MatLab (2010) e o MathCad (2010).

Concluída essa etapa, o passo seguinte foi implementar os modelos metabólicos de radionuclídeos de interesse do IPEN, conforme apresentado pela ICRP, e comparar os resultados do programa com os obtidos pela publicação.

Alguns radionuclídeos têm seu comportamento descrito por um simples modelo biocinético para sua atividade sistêmica, enquanto para outros é importante primeiramente determinar algumas informações adicionais como a distribuição ou a absorção de acordo com o tamanho da partícula. O modelo do trato respiratório humano foi criado para facilitar a aplicação de valores específicos para radionuclídeos inalados.

A ICRP 66 (1994) revisou este modelo pulmonar com informações mais recentes adicionando uma complexidade extra ao modelo, onde após a deposição do material, a absorção deste ocorre em um processo de dois estágios: a dissolução das partículas em um material que é absorvido pelo sangue, e a absorção direta do material solúvel que já se encontra desassociado das partículas.

\subsection{Urânio}

O radionuclídeo escolhido foi o Urânio $234\left({ }^{234} \mathrm{U}\right)$ com taxa de absorção do tipo S (Slow), em uma situação de incorporação aguda por inalação. Este é o tipo de inalação correspondente aos compostos $\mathrm{UO}_{2}$ e $\mathrm{U}_{3} \mathrm{O}_{8}$.

Um composto do tipo S implica na duplicação dos compartimentos do trato respiratório de forma que representem o mesmo compartimento com o material em estado transformado, conforme explicado na seção 3.1.1.

O valor do AMAD utilizado foi de $5 \mu \mathrm{m}$, o padrão recomendado para exposição ocupacional (ICRP 78, 1997). 
A ICRP apresenta os modelos biocinéticos específicos de cada radionuclídeo separados do modelo que representa o trato respiratório e o sistema gastrointestinal. Porém eles devem ser inseridos como um único modelo na atual versão do CBT, utilizando o plasma como compartimento de transferência entre eles.

O sistema compartimental criado para validação utilizou como base o modelo gastrointestinal da publicação 78 .

Apesar de se considerar uma incorporação por inalação, e não por ingestão, o material ainda pode alcançar o trato gastrointestinal, transferido por outros órgãos. Como explicado na seção 3.1.2, esta região é representada por quatro compartimentos, e quase todas suas taxas de transferência não variam de acordo com o material, exceção feita apenas a $\lambda_{B}$, que define única transferência entre essa região e o sangue, mostrado na equação (4).

O valor de $f 1$ relativo a cada elemento é dado no anexo específico deste radionuclídeo na ICRP 78. Para o urânio, este valor é 0,002, portanto:

$$
\lambda_{B}=\frac{(0,002 \cdot 6)}{(1-0,002)} \cong 0,012
$$

O modelo biocinético para a atividade sistêmica do radionuclídeo em questão contém outros 16 compartimentos. A fisiologia do modelo utilizado para o urânio é a mesma para estrôncio e rádio, e apenas os valores das taxas de transferência entre os compartimentos é que são alterados. A FIG. 15 apresenta o modelo biocinético destes 3 elementos, descrevendo em detalhes a sua cinética nos ossos, que é o principal ponto de retenção e deposição do material, e também considera retenções no fígado, rins e outros tecidos, assim como as rotas de excreção. 


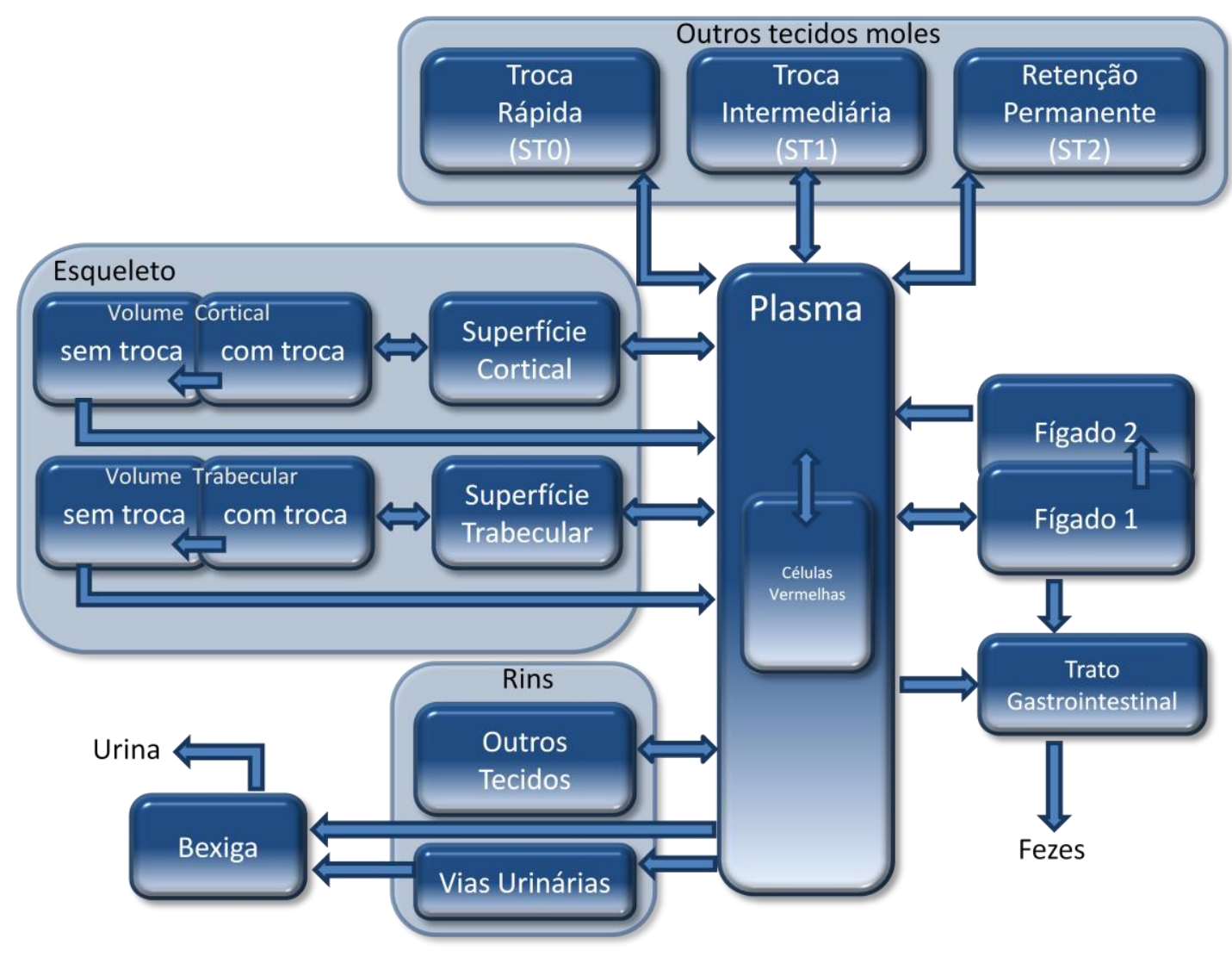

FIGURA 15 - Diagrama do modelo biocinético do urânio, adaptado da ICRP 78

Assim, o modelo compartimental completo para este caso, criado no CBT ficou com 48 compartimentos, que pode ser resolvido com um sistema de 48 equações diferenciais, o que seria extremamente trabalhoso para ser calculado sem recursos computacionais.

O SSID efetua o cálculo para um $\mathrm{t}=200$ dias em 14,8 segundos usando a implementação de Birchall (1989), e em 8,2 segundos usando o método de Runge-Kutta (Press et al., 1992).

O tempo necessário para a resolução de um modelo varia conforme a velocidade do processador utilizado, porém em geral o tempo para calcular um tempo $\mathrm{t}=200$ dias fica abaixo de 20 segundos.

Nos testes efetuados, resolver o modelo utilizando a implementação do método de Runge-Kutta demora cerca de $60 \%$ do tempo para a mesma resolução com a implementação do método de Birchall. A diferença nos resultados destes métodos ocorre a 
partir da décima casa decimal, portanto os resultados serão apresentados apenas como SSID independente do método de resolução utilizado.

A FIG. 14 exibe o gráfico com as curvas de retenção para os compartimentos plasma e estômago, e a curva de excreção para a urina, após a solução do sistema, para um tempo t de 700 dias.

Na TAB. 2 são apresentados os resultados dos primeiros dez dias após a incorporação calculados pelo programa SSID e os mesmos resultados apresentados pela ICRP 78 e por outros códigos computacionais semelhantes.

TABELA 2 - Comparação entre os resultados da ICRP, Humorap, AIDE e do SSID para o urânio

\begin{tabular}{|c|c|c|c|c|}
\hline $\begin{array}{c}\text { Dias após a } \\
\text { incorporação }\end{array}$ & ICRP 78 & Humorap & AIDE & SSID \\
\hline 1 & $7,00 \mathrm{E}-04$ & $7,04 \mathrm{E}-04$ & $7,04 \mathrm{E}-04$ & $7,04 \mathrm{E}-04$ \\
\hline 2 & $4,40 \mathrm{E}-05$ & $4,41 \mathrm{E}-05$ & $4,41 \mathrm{E}-05$ & $4,41 \mathrm{E}-05$ \\
\hline 3 & $2,60 \mathrm{E}-05$ & $2,60 \mathrm{E}-05$ & $2,60 \mathrm{E}-05$ & $2,60 \mathrm{E}-05$ \\
\hline 4 & $2,40 \mathrm{E}-05$ & $2,37 \mathrm{E}-05$ & $2,37 \mathrm{E}-05$ & $2,37 \mathrm{E}-05$ \\
\hline 5 & $2,20 \mathrm{E}-05$ & $2,20 \mathrm{E}-05$ & $2,20 \mathrm{E}-05$ & $2,20 \mathrm{E}-05$ \\
\hline 6 & $2,00 \mathrm{E}-05$ & $2,05 \mathrm{E}-05$ & $2,05 \mathrm{E}-05$ & $2,05 \mathrm{E}-05$ \\
\hline 7 & $1,90 \mathrm{E}-05$ & $1,92 \mathrm{E}-05$ & $1,92 \mathrm{E}-05$ & $1,92 \mathrm{E}-05$ \\
\hline 8 & $1,80 \mathrm{E}-05$ & $1,80 \mathrm{E}-05$ & $1,80 \mathrm{E}-05$ & $1,80 \mathrm{E}-05$ \\
\hline 9 & $1,70 \mathrm{E}-05$ & $1,70 \mathrm{E}-05$ & $1,70 \mathrm{E}-05$ & $1,70 \mathrm{E}-05$ \\
\hline 10 & $1,60 \mathrm{E}-05$ & $1,60 \mathrm{E}-05$ & $1,60 \mathrm{E}-05$ & $1,60 \mathrm{E}-05$ \\
\hline
\end{tabular}

Na TAB. 2 é observada uma diferença entre o SSID e a ICRP 78, que ocorre devido o arredondamento efetuado pela publicação. Ao comparar os valores com outros programas existentes, confirma-se a precisão do SSID.

\subsection{Iodo}

O segundo radionuclídeo a ser testado foi o Iodo $131\left({ }^{131} \mathrm{I}\right)$, que apresenta duas grandes diferenças com relação ao urânio testado anteriormente: uma meia-vida curta, de 8,04 dias, e presente em um composto cuja inalação é do tipo F (Fast). Isto implica uma grande mudança do modelo referente ao trato respiratório, pois, sendo o material de rápida absorção, não são necessárias as considerações sobre as partículas no estado transformado, o que reduz pela metade a quantidade de compartimentos nesta parte do modelo. 
Foi considerado neste estudo um AMAD de $5 \mu \mathrm{m}$, e o valor de $f 1$ fornecido pelo ICRP 78 para todos os compostos de iodo têm o mesmo valor 1.

O modelo sistêmico fornecido pela ICRP 78 para o iodo tem apenas 3 compartimentos, conforme observado na FIG. 16.

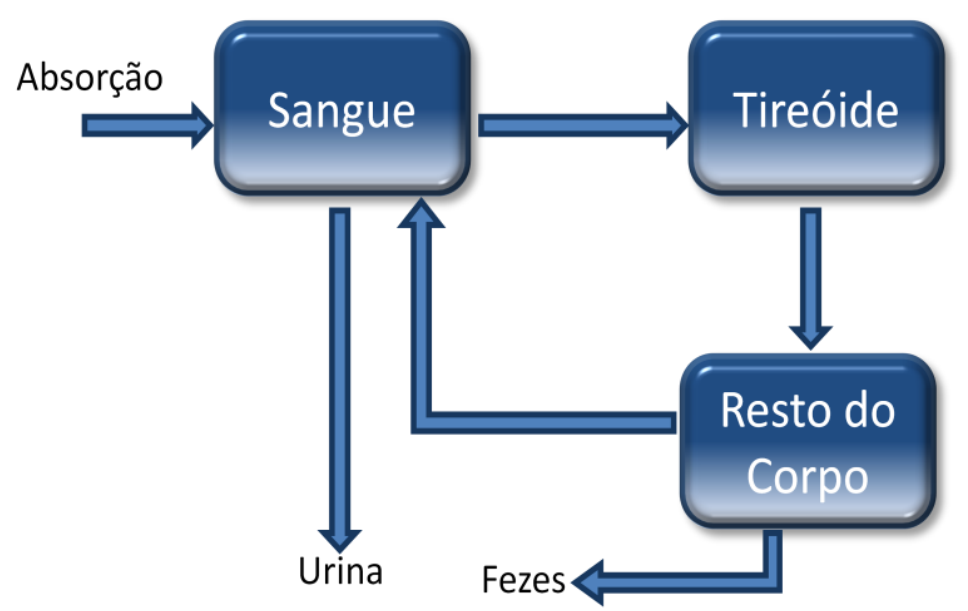

FIGURA 16 - Modelo sistêmico para o metabolismo do iodo, adaptado da ICRP 78

Com um sistema respiratório que não precisa de um espelhamento de seus compartimentos, e um modelo biocinético mais simples, o modelo completo criado no CBT possui apenas 21 compartimentos, e o SSID o resolve para um tempo (t) de 200 dias, em 1,5 segundos utilizando o método de Birchall e 0,9 segundos com Runge-Kutta.

A FIG.17 apresenta as curvas de retenção geradas pelo programa, dos compartimentos relativos ao sangue e à tireóide do indivíduo. 


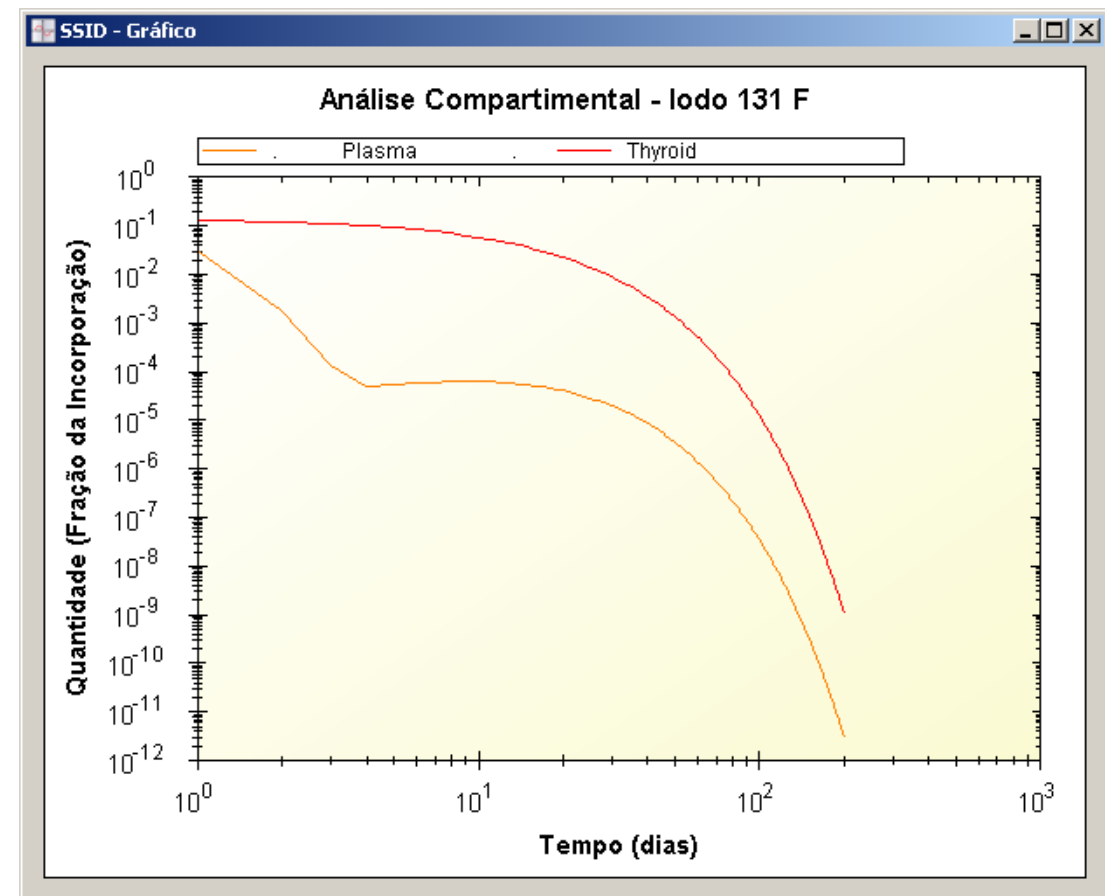

FIGURA 17 - Curvas de retenção do sangue e da tireóide para o Iodo, gerado pelo SSID

A TAB. 3 faz a comparação entre os valores dos primeiros dez dias seguintes à incorporação, calculados pelo SSID na tireóide e os apresentados pela ICRP 78, e apresentados na figura 18 .

Tabela 3 - Comparação entre os resultados da ICRP e do SSID para o Iodo

\begin{tabular}{|c|c|c|c|}
\hline $\begin{array}{c}\text { Dias após a } \\
\text { incorporação }\end{array}$ & ICRP & SSID & $\begin{array}{c}\text { Variação } \\
\text { Percentual }\end{array}$ \\
\hline 1 & $1,2 \mathrm{E}-01$ & $1,2 \mathrm{E}-01$ & $0,00 \mathrm{E}+00$ \\
\hline 2 & $1,2 \mathrm{E}-01$ & $1,2 \mathrm{E}-01$ & $0,00 \mathrm{E}+00$ \\
\hline 3 & $1,1 \mathrm{E}-01$ & $1,1 \mathrm{E}-01$ & $0,00 \mathrm{E}+00$ \\
\hline 4 & $9,9 \mathrm{E}-02$ & $9,9 \mathrm{E}-02$ & $0,00 \mathrm{E}+00$ \\
\hline 5 & $9,0 \mathrm{E}-02$ & $9,0 \mathrm{E}-02$ & $0,00 \mathrm{E}+00$ \\
\hline 6 & $8,2 \mathrm{E}-02$ & $8,2 \mathrm{E}-02$ & $0,00 \mathrm{E}+00$ \\
\hline 7 & $7,4 \mathrm{E}-02$ & $7,5 \mathrm{E}-02$ & $8,20 \mathrm{E}-01$ \\
\hline 8 & $6,8 \mathrm{E}-02$ & $6,8 \mathrm{E}-02$ & $0,00 \mathrm{E}+00$ \\
\hline 9 & $6,2 \mathrm{E}-02$ & $6,2 \mathrm{E}-02$ & $0,00 \mathrm{E}+00$ \\
\hline 10 & $5,6 \mathrm{E}-02$ & $5,6 \mathrm{E}-02$ & $0,00 \mathrm{E}+00$ \\
\hline
\end{tabular}

Assim como no urânio, os resultados obtidos são idênticos aos da ICRP, exceção feita ao sétimo dia de incorporação, onde uma diferença mínima observada parece ocorrer por diferenças de arredondamento. A ICRP não fornece esses valores com uma precisão maior para que uma comparação mais precisa pudesse ser feita. 


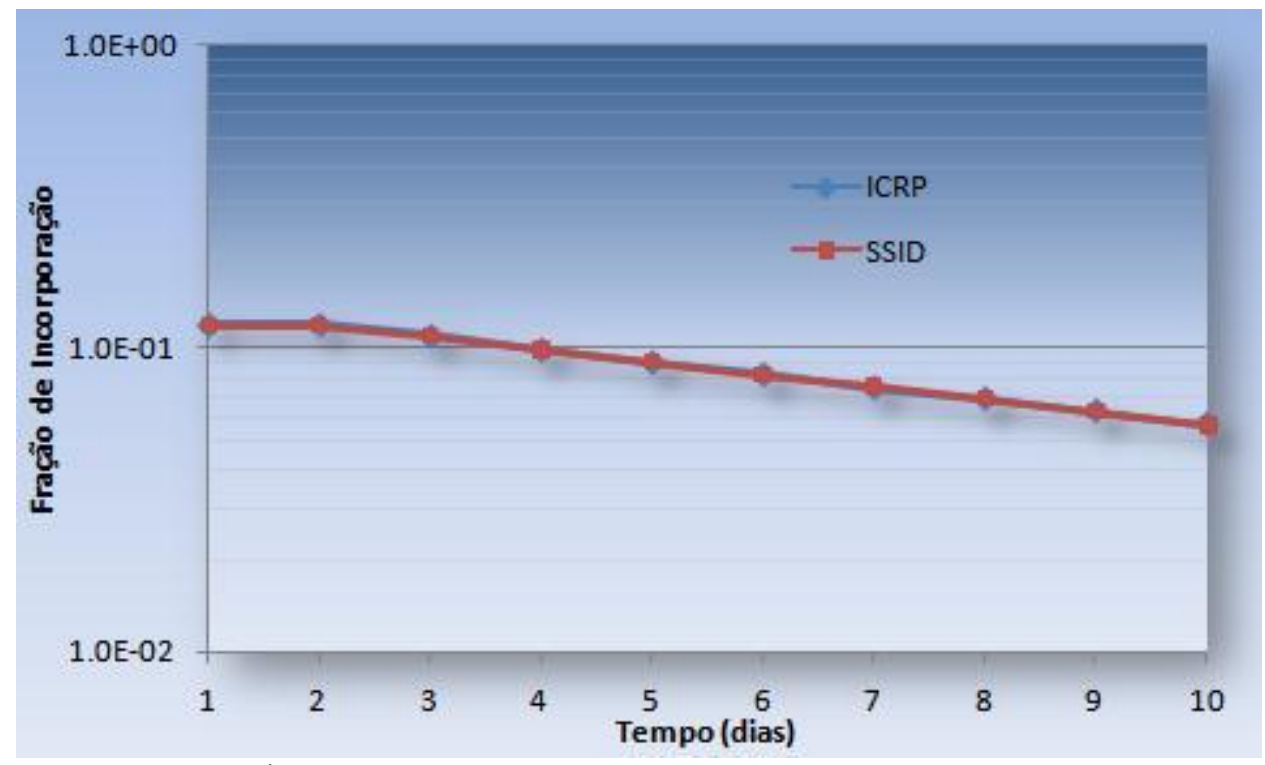

FIGURA 18 - Gráfico comparativo entre os resultados da ICRP e o SSID para o Iodo

\subsection{Tório}

O próximo radionuclídeo de interesse do IPEN a ser inserido no sistema foi o tório, que tem como principais pontos de deposição o fígado e o esqueleto. Os valores de transferência do modelo biocinético para o tório também foram obtidos da ICRP 78.

O modelo publicado pela primeira vez na ICRP 67 apresenta a cinética dos elementos tório, plutônio, amerício, cúrio e netúnio (FIG. 19). Os parâmetros específicos para o tório foram publicados em 1995, na ICRP 69. Para alguns destes materiais, o fígado pode ser visto como um único compartimento, enquanto outros, como o tório, apresenta um segundo compartimento para representar diferentes liberações do material absorvido. 


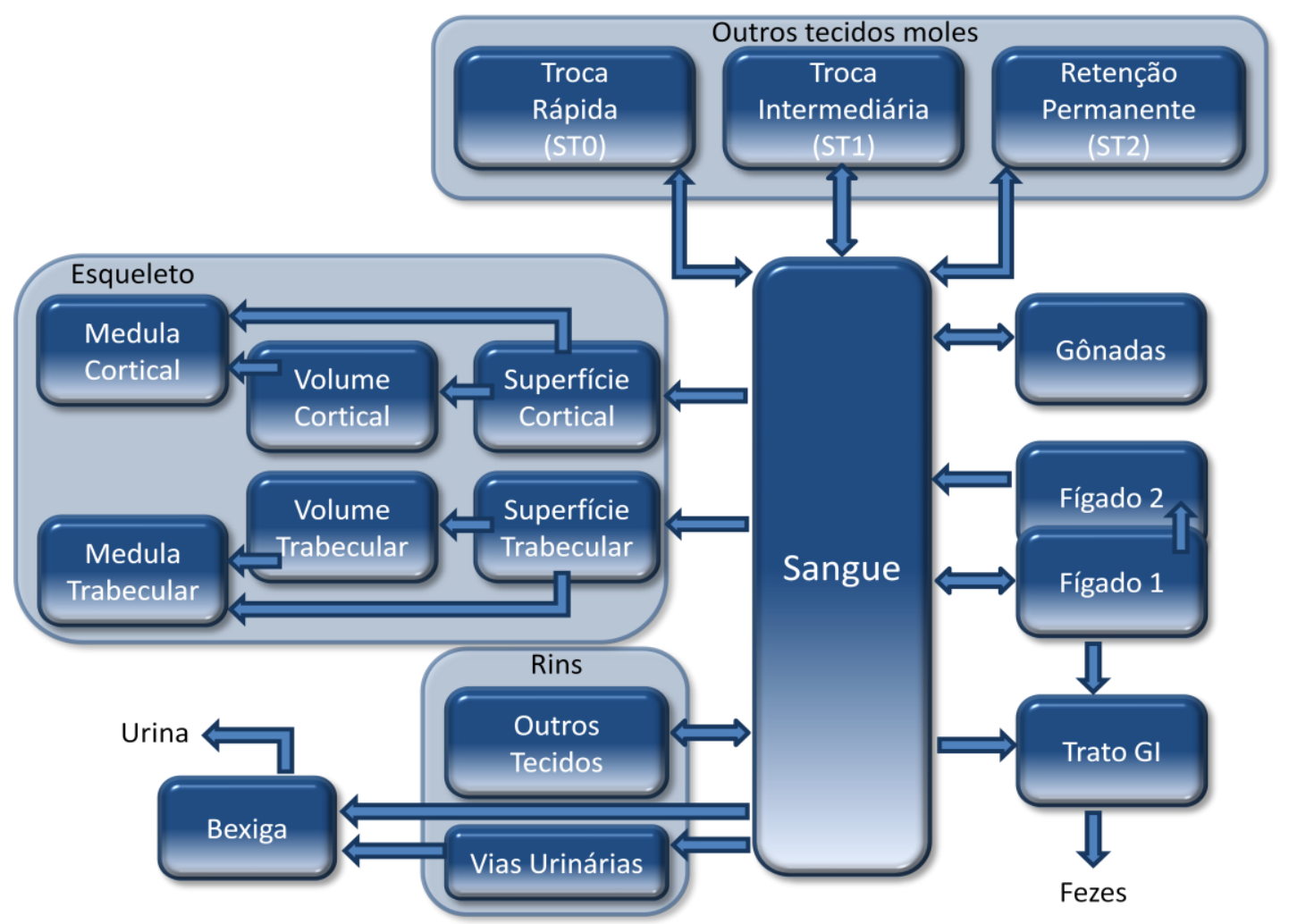

FIGURA 19 - Diagrama do modelo biocinético para tório, plutônio, amerício, cúrio e netúnio. Adaptado da ICRP 78

Considerou-se um caso de inalação única de $\mathrm{Th}^{228}$ presente em um composto do tipo $\mathrm{S}$ (Slow) e com o AMAD padrão de $5 \mu \mathrm{m}$. Neste ponto do trabalho, com o CBT devidamente consolidado, constatou-se a facilidade em inserir um novo modelo, usando como base o modelo já existente do urânio e apenas alterando os poucos compartimentos diferentes, e as taxas de transferência de todos eles.

O valor de $f 1$ utilizado foi de 2,0E-04, para compostos como óxidos e hidróxidos. A meia-vida do Tório 228 é de 1,91 anos. O CBT utiliza apenas a unidade dias para meia-vida, devendo assim informar o valor 1,91 x 365 ao criar o modelo compartimental.

O modelo foi resolvido calculando as frações de incorporações para 200 dias, em 20,3 segundos usando o método de resolução de Birchall e em 5,6 segundos com Runge-Kutta. Os valores obtidos são idênticos aos valores de referência publicados pela ICRP 78. 
A FIG. 20 apresenta o gráfico gerado pelo programa após a resolução de um modelo para o Tório 228, com os valores de retenção do plasma e do estômago, e os valores de excreção da urina, calculados até 200 dias.

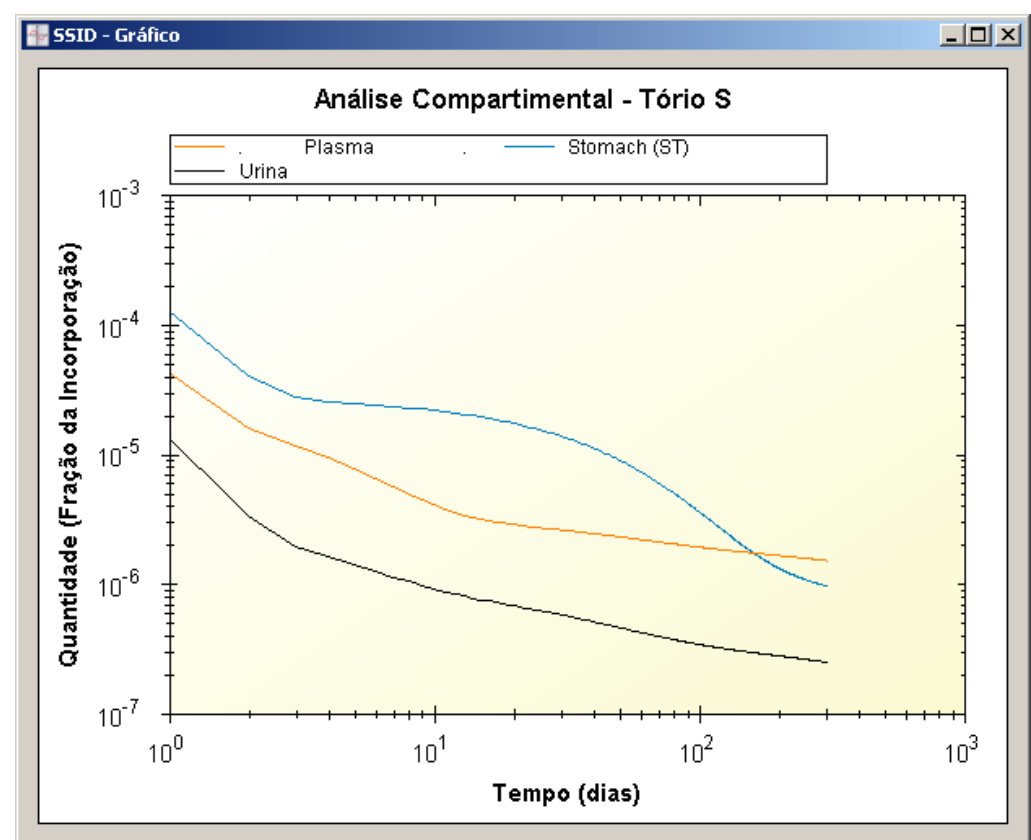

FIGURA 20 - Gráfico com os valores de retenção e excreção do Tório 228 gerado pelo SSID 


\section{CONCLUSÃO}

Os resultados obtidos comprovam a eficiência do programa para resolver modelos compartimentais por meio de sistemas de equações diferenciais, alcançando assim o objetivo do trabalho.

Durante a implementação dos modelos estudados, verificou-se a facilidade que o programa agrega ao processo de criação do modelo compartimental, e eventuais ajustes em sua estrutura.

A utilização do CBT como uma ferramenta genérica de construção de modelos compartimentais, com suas características de troca de dados com outras aplicações pelo padrão XML, possibilita o desenvolvimento de outros códigos computacionais que substituam o SSID, com foco específico em outras aplicações, como no estudo da dispersão de poluentes em um ambiente, ou mesmo na farmacocinética, que também faz uso dos modelos compartimentais para avaliar o tempo de retenção de uma droga no organismo.

Com todas as funcionalidades pretendidas completamente implementadas, o programa desenvolvido se tornará uma ferramenta de grande auxílio ao grupo de dosimetria interna do IPEN.

\subsection{Sugestões para trabalhos futuros}

Durante o desenvolvimento do trabalho, foram observadas algumas necessidades do programa de monitoração da proteção radiológica do IPEN que poderiam ser sanadas com uma integração deste programa com um módulo para o cálculo de dose e armazenamento de dados de trabalhadores, como o desenvolvido por Lima (2007).

Um aprimoramento do código SSID poderia solicitar como parâmetros de entrada os valores obtidos das análises de excretas do indivíduo, e apontar qual curva de excreção melhor se ajusta a estes pontos. 
Implementações computacionais de outros métodos matemáticos para a solução de equações diferenciais podem ser adicionadas ao SSID, permitindo estudos mais detalhados sobre a eficiência e a eficácia destas, em precisão e tempo de processamento. 


\section{APÊNDICE A - Exemplo de XML gerado pelo CBT}

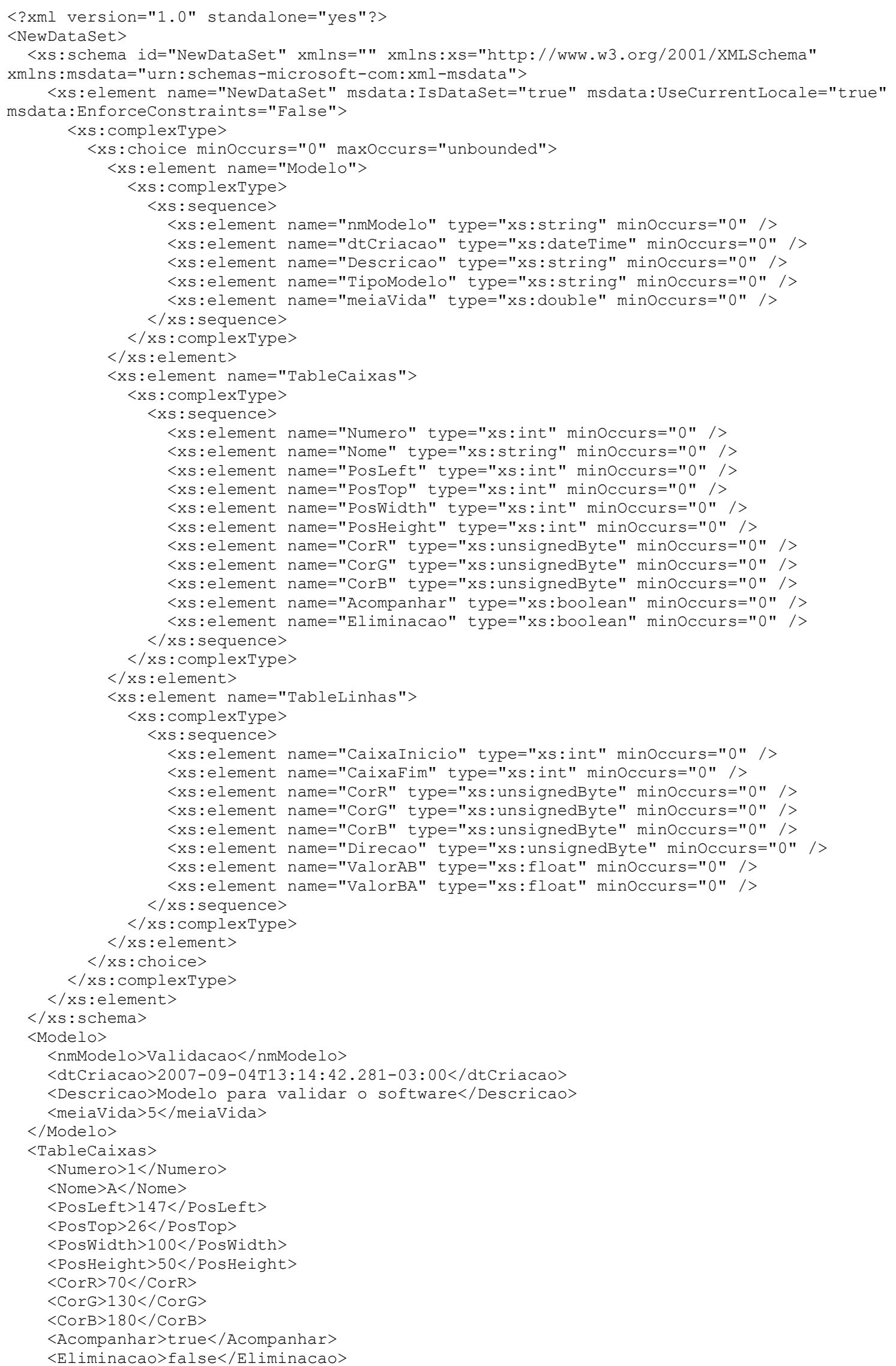




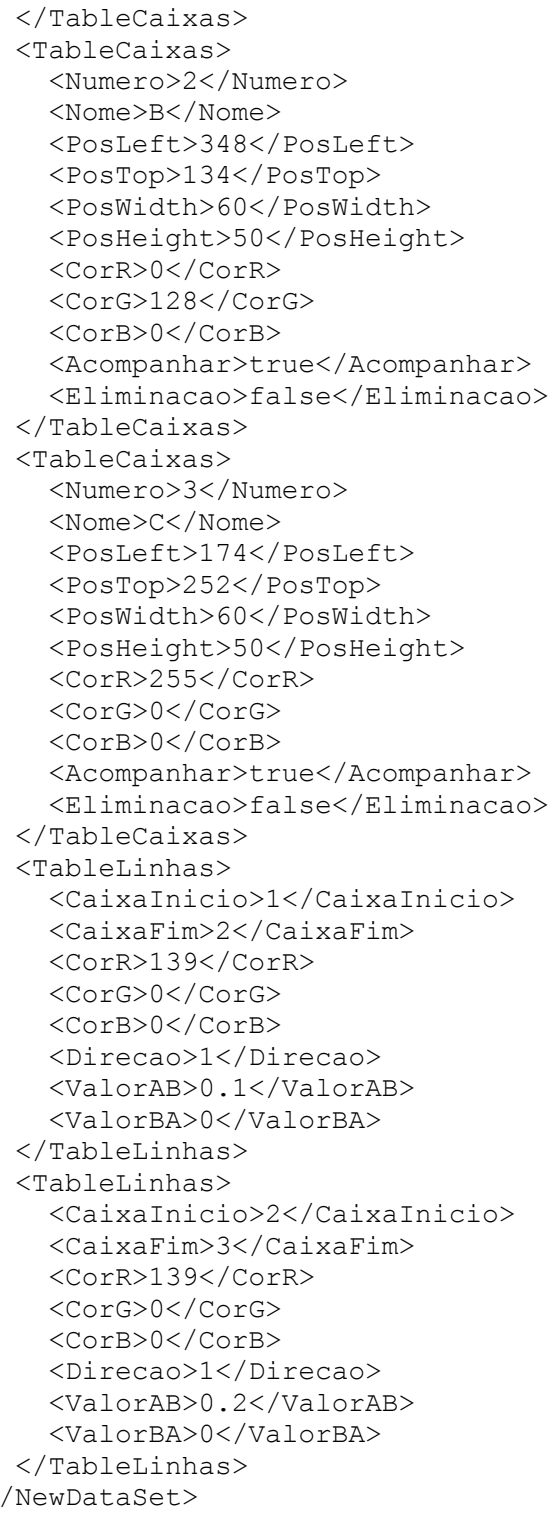




\section{APÊNDICE B - Exemplo de relatório gerado pelo SSID}

\section{Relatório}

Modelo: Tório S

Gerado em: 27/01/2011 15:25

Modelo de Tório Slow Amad 5

Resolvido pelo método: Runge-Kutta 45

\begin{tabular}{|c|c|c|c|c|}
\hline Tempo & . Plasma . & Stomach (ST) & Urina & Total \\
\hline 0 & $0.0000000000 e+000$ & $0.0000000000 e+000$ & $0.0000000000 e+000$ & $4.8200000000 \mathrm{e}-001$ \\
\hline 1 & $4.2347489962 \mathrm{e}-005$ & $1.2742861989 \mathrm{e}-004$ & $1.3470130273 e-005$ & $4.8193463731 \mathrm{e}-001$ \\
\hline 2 & $1.5977442699 \mathrm{e}-005$ & $3.9965169428 \mathrm{e}-005$ & $3.3137644197 e-006$ & $4.8186928348 \mathrm{e}-001$ \\
\hline 3 & $1.1689574715 \mathrm{e}-005$ & $2.7641975352 \mathrm{e}-005$ & $1.9364200072 \mathrm{e}-006$ & $4.8180393852 \mathrm{e}-001$ \\
\hline 4 & $9.3914974100 \mathrm{e}-006$ & $2.5453780234 \mathrm{e}-005$ & $1.6091193772 \mathrm{e}-006$ & $4.8173860241 \mathrm{e}-001$ \\
\hline 5 & $7.7392193178 \mathrm{e}-006$ & $2.4645703994 \mathrm{e}-005$ & $1.4031951231 \mathrm{e}-006$ & $4.8167327517 e-001$ \\
\hline 6 & $6.5215105827 e-006$ & $2.4042247776 \mathrm{e}-005$ & $1.2496036996 \mathrm{e}-006$ & $4.8160795678 \mathrm{e}-001$ \\
\hline 7 & $5.6140238739 \mathrm{e}-006$ & $2.3474169913 \mathrm{e}-005$ & $1.1324807196 \mathrm{e}-006$ & $4.8154264726 \mathrm{e}-001$ \\
\hline 8 & $4.9418339310 \mathrm{e}-006$ & 2.2928409877e-005 & $1.0422028398 \mathrm{e}-006$ & 4.8147734659e-001 \\
\hline 9 & $4.4358794283 e-006$ & 2.2392472848e-005 & $9.7181528358 \mathrm{e}-007$ & 4.8141205477e-001 \\
\hline 10 & $4.0596590329 \mathrm{e}-006$ & $2.1874862207 e-005$ & $9.1620477340 \mathrm{e}-007$ & $4.8134677181 \mathrm{e}-001$ \\
\hline 11 & $3.7719412640 \mathrm{e}-006$ & $2.1365913221 \mathrm{e}-005$ & $8.7160217830 \mathrm{e}-007$ & $4.8128149770 \mathrm{e}-001$ \\
\hline 12 & $3.5566011913 \mathrm{e}-006$ & $2.0874387808 \mathrm{e}-005$ & $8.3522905005 e-007$ & $4.8121623244 \mathrm{e}-001$ \\
\hline 13 & $3.3878441639 \mathrm{e}-006$ & $2.0391074244 \mathrm{e}-005$ & $8.0503436694 \mathrm{e}-007$ & $4.8115097604 \mathrm{e}-001$ \\
\hline 14 & $3.2601030569 \mathrm{e}-006$ & $1.9924139255 \mathrm{e}-005$ & $7.7950464740 \mathrm{e}-007$ & $4.8108572848 \mathrm{e}-001$ \\
\hline 15 & $3.1565024067 \mathrm{e}-006$ & $1.9465217397 \mathrm{e}-005$ & $7.5752046609 \mathrm{e}-007$ & $4.8102048977 e-001$ \\
\hline 16 & $3.0766061191 \mathrm{e}-006$ & $1.9021496076 \mathrm{e}-005$ & $7.3825438154 \mathrm{e}-007$ & $4.8095525991 \mathrm{e}-001$ \\
\hline 17 & $3.0089930063 e-006$ & $1.8585770375 \mathrm{e}-005$ & $7.2109243077 \mathrm{e}-007$ & $4.8089003889 \mathrm{e}-001$ \\
\hline 18 & $2.9553615110 \mathrm{e}-006$ & $1.8163980870 \mathrm{e}-005$ & $7.0557946723 e-007$ & $4.8082482672 \mathrm{e}-001$ \\
\hline 19 & $2.9079178494 \mathrm{e}-006$ & $1.7750310244 \mathrm{e}-005$ & $6.9137603715 e-007$ & $4.8075962339 \mathrm{e}-001$ \\
\hline 20 & $2.8688205561 \mathrm{e}-006$ & $1.7349250172 \mathrm{e}-005$ & $6.7822922903 e-007$ & $4.8069442890 \mathrm{e}-001$ \\
\hline 21 & $2.8329585919 \mathrm{e}-006$ & $1.6956544285 \mathrm{e}-005$ & $6.6594890907 \mathrm{e}-007$ & $4.8062924326 \mathrm{e}-001$ \\
\hline 22 & $2.8020089610 \mathrm{e}-006$ & $1.6575087114 \mathrm{e}-005$ & $6.5439220353 e-007$ & $4.8056406645 \mathrm{e}-001$ \\
\hline 23 & $2.7730547659 \mathrm{e}-006$ & $1.6202302882 \mathrm{e}-005$ & $6.4345042176 \mathrm{e}-007$ & $4.8049889848 \mathrm{e}-001$ \\
\hline 24 & $2.7467689278 \mathrm{e}-006$ & $1.5839394524 \mathrm{e}-005$ & $6.3304072679 e-007$ & $4.8043373935 e-001$ \\
\hline 25 & $2.7215726207 e-006$ & $1.5484869441 \mathrm{e}-005$ & $6.2309903175 \mathrm{e}-007$ & $4.8036858906 \mathrm{e}-001$ \\
\hline
\end{tabular}

Tempo de processamento: 00:00:00:500 


\section{REFERÊNCIAS BIBLIOGRÁFICAS}

ANSOBORLO, E., BÉRARD, P., ECKERMAN, K., BERKOVSKI, V., BIRCHALL, A., FRY, F., GUILMETTE, R., MILLER, G., ISHIGURE, N., LIPSZTEIN, J., NOSSKE, D. Review of methods and computer codes for interpretation of bioassy data. Radiat. Prot. Dosim. v 105, p 341-346, 2003.

BERTELLI, L.; MELO, D. R.; LIPSZTEIN, J.; CRUZ-SUAREZ, R.. AIDE: Internal Dosimetry Software. Radiat Prot. Dosimetry, v. 130, p. 358-367, 2008.

BIRCHALL, A. \& JAMES, A. C. A microcomputer algorithm for solving first-order compartmental models involving recycling. - Health Phys., 56(6):857-868, 1989.

BIRCHALL, A., JARVIS, N. S., PEACE, M. S., RIDDELL A. E. BATTERSBY, W. P. The IMBA suite: integrated modules for bioassay analysis. Radiat. Prot. Dosim. v. 79, p. 107-110, 1998.

BIRCHALL, A., PUNCHER, M., JAMES, A. C., MARSH, J. W., JARVIS, N. S., PEACE, M. S, DAVIS, K., KING, D. J. IMBA-EXPERT: internal dosimetry made simple. Radiat. Prot. Dosim. v. 105, p. 421-426, 2003.

BIRCHALL, A., PUNCHER, M., MARSH, J. W., DAVIS, K., BAILEY, M. R., JARVIS, N. S., A. D. PEACH, DORRIAN, M. D., JAMES, A. C., IMBA Professional plus: A flexible approach to internal dosimetry. Radiat. Prot. Dosim. v. 125, n. 1-4, p. 194-197, 2006

COMISSÃO NACIONAL DE ENERGIA NUCLEAR (CNEN-NN-3.01). Diretrizes básicas de proteção radiológica, Rio de Janeiro, 2005.

DOTNUMERICS. Computação numérica para plataforma Microsoft .NET. Apresenta uma biblioteca para computação numérica englobando resolução de sistemas de equações diferenciais. Disponível em: 〈http://www.dotnumerics.com〉. Acesso em: 01 ago. 2010.

FRAPPE, F. N., RANNOU, A. General solution of the ICRP 66 model and its application to given radionuclides. Radiat. Prot. Dosim. v. 79, n 1-4, p. 29-32, 1998.

INTERNATIONAL COMMISSION ON RADIOLOGICAL PROTECTION (ICRP-26). Recommendations of the international commission on radiological protection Publication 26, Pergamon Press, Oxford, 1977.

INTERNATIONAL COMMISION ON RADIOLOGICAL PROTECTION (ICRP-30). Limits for intakes of radionuclides by workers - Publication 30, Pergamon Press, Oxford, 1979.

INTERNATIONAL COMMISSION ON RADIOLOGICAL PROTECTION (ICRP-35). General principles for monitoring of radiation protection of workers - Publication 35 , Pergamon Press, Oxford, 1982. 
INTERNATIONAL COMMISION ON RADIOLOGICAL PROTECTION (ICRP-54). Individual monitoring for intakes of radionuclides by workers: design and interpretation - Publication 54, Pergamon Press, Oxford, 1988.

INTERNATIONAL COMMISSION ON RADIOLOGICAL PROTECTION (ICRP-56). Age-dependent doses to members of the public from intake of radionuclides- Part 1 Publicaton 56, Pergamon Press, Oxford, 1990.

INTERNATIONAL COMMISSION ON RADIOLOGICAL PROTECTION (ICRP-60). Recommendations of the international commission on radiological protection 1990 Publication 60, Pergamon Press, Oxford, 1991.

INTERNATIONAL COMMISSION ON RADIOLOGICAL PROTECTION (ICRP61). Annual limits for intakes of radionuclides by workers based on the 1990 Recommendations - Publication 61, Pergamon Press, Oxford, 1991.

INTERNATIONAL COMMISION ON RADIOLOGICAL PROTECTION (ICRP-66). The human respiratory tract model - Publication 66, Pergamon Press, Oxford, 1994.

INTERNATIONAL COMMISSION ON RADIOLOGICAL PROTECTION (ICRP-67). Age-dependent doses to members of the public from intake of radionuclides- Part 2 Ingestion dose coefficients - Publication 67, Pergamon Press, Oxford, 1994.

INTERNATIONAL COMMISSION ON RADIOLOGICAL PROTECTION (ICRP-68). Dose coefficients for intakes of radionuclides by workers - Publication 68, Pergamon Press, Oxford, 1994.

INTERNATIONAL COMMISSION ON RADIOLOGICAL PROTECTION (ICRP-69). Age-dependent doses to members of the public from intake of radionuclides-Part 3. Ingestion dose coefficients - Publication 69, Oxford, 1995.

INTERNATIONAL COMMISSION ON RADIOLOGICAL PROTECTION (ICRP-75). General principles for the radiation protection of workers - Publication 75, Pergamon Press, Oxford, 1997.

INTERNATIONAL COMMISION ON RADIOLOGICAL PROTECTION (ICRP-78). Individual monitoring for internal exposure of workers - Replacement of publication 54 - Publication 78, Pergamon Press, Oxford, 1997.

INTERNATIONAL COMMISION ON RADIOLOGICAL PROTECTION (ICRP-100). Human alimentary tract model for radiological protection- Publication 100, Pergamon Press, Oxford, 2007.

INTERNATIONAL COMMISSION ON RADIOLOGICAL PROTECTION. Task Group 21 (INDOS) Internal Dosimetry. Disponível em <http://www.icrp.org/icrp_group.asp?id=36>. Acesso em: 01 mar. 2011.

ISHIGURE, N., MATSUMOTO, M., NAKANO, T., ENOMOTO, H. Development of software for internal dose calculation from bioassay measurements. Radiat. Prot. Dosim. V 109, n 3, p 235-242, 2004. 
JARVIS, N. S., BIRCHALL, A. LUDEP 1.0, a personal computer program to implement the new ICRP respiratory tract model. Radiat. Prot. Dosim. V 53, n 1-4, p 191-193, 1994.

LIMA, V. R. Desenvolvimento de um sistema baseado na web para suporte ao programa de monitoração individual interna do Ipen. 2007. Dissertação (Mestrado) Instituto de Pesquisas Energéticas e Nucleares, São Paulo.

LOURENÇO, M. C. Análise Paramétrica de Modelos Biocinéticos Utilizados em Dosimetria Interna. 1997. Tese (Doutorado) - Universidade Federal do Rio de Janeiro, Instituto de Biofísica, Rio de Janeiro.

MATHCAD, versão 14: software para cálculo numérico. [S.1.] PTC, 2010.

MATLAB, versão 2010: software para cálculo numérico. [S.1.] Mathworks, 2010.

PRESS, W. H.; TEUKOLSKY, S. A.; VETTERLING, W. T.; FLANNERY, B. P. Numerical Recipes in Fortran - The Art of Scientific Computing. 2. ed. New York: Cambridge University Press, 1992.

RESCIGNO, A. On the use of pharmacokinetic models. Phys. Med. Biol., v. 49, p. 46574676, 2004.

RODRIGUES Jr., O. Aplicação de Modelos Metabólicos para a Determinação de Funções de Excreção e Retenção. 1994. Dissertação (Mestrado) - Instituto de Pesquisas Energéticas e Nucleares, São Paulo.

SANCHES, M. P. Identificação e análise dos principais radionuclídeos que potencialmente contribuem para a dose interna dos trabalhadores do centro de radiofarmácia. 2004. Dissertação (Mestrado) - Instituto de Pesquisas Energéticas e Nucleares, São Paulo.

SANCHEZ, G.; LOPEZ-FIDALGO, J. Mathematical Techniques for Solving Analytically Large Compartmental Systems. Health Physics, v. 85, 2003.

STATHER, J. W.: The Developement of Protection Standards for Intakes of Radionuclides (1995-2005). Radiation Protection Dosimetry, v. 109 No. 4, 2004.

TIOBE. Programming Community Index. TIOBE Programming Community Index for May 2011. Disponível em <http://www.tiobe.com/index.php/content/paperinfo/tpci/index.html>. Acesso em: 01 ago. 2010. 